\title{
Six-dimensional phase space preservation in a terahertz-driven multistage dielectric-lined rectangular waveguide accelerator
}

\author{
Öznur Apsimon $\odot,{ }^{1,2, * \dagger}$ Graeme Burt ${ }^{1,2}$ Robert B. Appleby® ${ }^{1,3}$ Robert J. Apsimon $\odot,{ }^{1,2}$ \\ Darren M. Graham $\oplus^{1,4}$ and Steven P. Jamison $\oplus^{1,5}$ \\ ${ }^{1}$ The Cockcroft Institute, Sci-Tech Daresbury, Keckwick Lane, Daresbury, \\ Warrington WA4 4AD, United Kingdom \\ ${ }^{2}$ Department of Engineering, Lancaster University, Bailrigg, Lancaster LAl 4YW, United Kingdom \\ ${ }^{3}$ Department of Physics and Astronomy, The University of Manchester, \\ Oxford Road, Manchester M13 9PL, United Kingdom \\ ${ }^{4}$ Department of Physics and Astronomy \& Photon Science Institute, The University of Manchester, \\ Oxford Road, Manchester M13 9PL, United Kingdom \\ ${ }^{5}$ Department of Physics, Lancaster University, Bailrigg, Lancaster LA1 4YW, United Kingdom
}

(Received 7 May 2021; accepted 4 November 2021; published 7 December 2021)

\begin{abstract}
Staged acceleration, driven by terahertz $(\mathrm{THz})$ frequency radiation pulses in a lattice with alternating orientation dielectric-lined waveguides and intervening matching optics, is shown to mitigate transverse emittance and energy spread growth, opening a route to multistage $\mathrm{THz}$ linacs. Decomposition of the longitudinal $\mathrm{THz}$ field into the multipolar components reveals a quadrupole field component with strong radial dependence. As such, it induces a transverse energy correlation in the beam during acceleration due to the large variation in the electric field with radius and azimuthal position of the electrons. An alternating orientation of stages separated by a matching section provides a compensation of transverse energy spread correlation induced in the beam during its interaction with the $\mathrm{THz}$ field. Furthermore, the monopolar component of the accelerating $\mathrm{LSM}_{11}$ mode was found to be constant with respect to transverse position, entailing zero monopolar transverse voltage and preventing emittance growth, unlike conventional radiofrequency structures. We demonstrate in a rectangular dielectric-lined waveguide structure that, when used for the acceleration of relativistic electrons, the slice transverse emittance is conserved and the growth in the slice energy spread is reduced by $70 \%-80 \%$ simultaneously over a system of two stages, each providing an interaction length of $4 \mathrm{~mm}$ and an energy gain of up to $2 \mathrm{MeV}$.
\end{abstract}

DOI: $10.1103 /$ PhysRevAccelBeams.24.121303

\section{INTRODUCTION}

The next generation of free electron lasers and particle colliders will require injectors that can deliver high-quality beams with bunch durations far shorter than what are currently achievable with higher accelerating gradients than currently available. To achieve this, high frequency acceleration systems are required but laser and plasma accelerators have so far failed to deliver the required beam quality. While plasma based novel technologies have been able to generate very high gradients, they have so far been unable to generate beams with the quality, control-ability

\footnotetext{
*oznur.apsimon@liverpool.ac.uk

Present address: The University of Liverpool and the Cockcroft Institute.

Published by the American Physical Society under the terms of the Creative Commons Attribution 4.0 International license. Further distribution of this work must maintain attribution to the author(s) and the published article's title, journal citation, and DOI.
}

and stability required for most accelerator applications. A common challenge for all novel acceleration methods is preserving the beam quality while achieving high-energy gain. Plasma acceleration faces emittance and energy spread growth due to transverse fields almost as large as longitudinal fields [1], scattering by plasma electrons and ions [2], and field oscillations with much smaller periods compared to conventional radio-frequency technologies. Hence, other novel accelerator concepts are required to meet the future demand for compact particle accelerators with high-quality beams.

There are a number of promising candidates for compact particle accelerators, including dielectric wakefield accelerators [3], mm-wave accelerating structures, $[4,5]$ beamdriven plasma wakefield accelerators, [6] and terahertz (THz)-driven accelerators [7]. THz-driven accelerators offer a potential solution due to their larger size compared to optical accelerators and shorter wavelength compared to conventional radio-frequency accelerators. The latter allows the bunch length to be reduced below $100 \mathrm{fs}$ without the need for velocity bunching in cavities or magnetic 
compression commonly implemented alongside with radiofrequency technology.

The realization of laser-driven high-field THz sources [8] led to a number of concepts being proposed for the use of $\mathrm{THz}$ radiation for the acceleration and manipulation of electron beams, including interactions with two counterpropagating $\mathrm{THz}$ pulses $[9,10]$, in circular dielectric-lined waveguides [11,12], using a subluminal traveling terahertz source [13] and high-power narrow-band $\mathrm{THz}$ generation [14]. A concept has also been proposed for using an evanescent-wave scheme to accelerate protons [15]. THz acceleration has been experimentally demonstrated at nonrelativistic [16-19] and, more recently, relativistic beam energies, using either a laser-driven $\mathrm{THz}$ source [7] or a coherent-transition-radiation-based $\mathrm{THz}$ source [20]. Frontier studies focus on the stability and scalability by means of multistaging [21].

The first demonstration of laser-driven $\mathrm{THz}$ acceleration of relativistic electrons was conducted in 2019 using the Compact Linear Accelerator for Research and Applications (CLARA) test facility at Daresbury Laboratory [7]. This experiment used a dielectric-lined rectangular waveguide structure driven by narrow-band, frequency-tunable, polarization-tailored $\mathrm{THz}$ pulses and demonstrated up to $10 \mathrm{keV}$ acceleration of a 2 ps (subcycle) bunch with an initial energy of $35 \mathrm{MeV}$. Consequently, it marked a key milestone on the path to whole-bunch linear acceleration of subpicosecond electron beams with multistaged concepts capable of preserving beam quality.

The control and preservation of beam quality is central to the success of future THz-driven accelerators. But solutions to implement them in a scheme that preserves beam properties has yet to be studied in detail. The figure of merit for beam quality is six-dimensional (6D) phase-space brightness which includes both transverse emittance and energy spread. For a beam with a finite longitudinal size, both quantities can be defined for a thin longitudinal slice or for the entire bunch of a beam as a projection of all slices. In some cases, where nonlinear forces are exerted on the beam, such as space charge defocusing or the time variation of an accelerating field across a bunch, the projected quantities can be large despite the fact that each slice has a high 6D brightness. Consequently, it is relevant to distinguish both definitions and characterize the 6D phase space for each, especially for applications such as free electron lasers where slice properties are more relevant due to the microbunching process dominating the radiation emission characteristics. Previously, synchronous acceleration of nonrelativistic electrons in a tapered dielectric-lined waveguide structure [22], the effect of the longitudinal focus position and phase stability on the beam dynamics [23] and compensation of nonlinear energy chirp due to radio-frequency field curvature in corrugated structures [24] have been studied.

In this work, we present preservation of 6D phase space, entailing both a low emittance and low-energy spread simultaneously, for synchronous acceleration of relativistic electrons in a rectangular dielectric lined waveguide structure. In a multistage arrangement, it is important to have all stages synchronous with the electron beam and ideally at the same frequency to simplify the $\mathrm{THz}$ sources. This requires some degree of tunability in the accelerating structure, which is easily achieved with rectangular dielectric lined waveguides as the gap can be adjusted. In addition, these structures offer flexibility after manufacturing for frequency fine-tuning by means of spacers or trimming to vary the gap. It is also possible to taper a rectangular waveguide to match to a particular beam velocity, although this is less important for fully relativistic cases, whereas in a circular waveguide only dielectric thickness and metal radius can be varied for a given dielectric material that renders matching optimization impossible as aperture is normally fixed, and the dielectric thickness is determined by the quarter-wave variation in dielectric. However, a rectangular waveguide enables precise synchronism via optimization of width and height of the aperture and $\mathrm{THz}$ frequency. However, rectangular structures also have complex field variations which could increase emittance or energy spread. In this paper, we demonstrate that this effect can be completely canceled out opening the door to tunable DLW that are capable of preserving emittance.

The layout of this paper is as follows. In Sec. II we introduce the analysis of the multipole field components decomposed from the longitudinal THz field at $\mathrm{LSM}_{11}$ mode. In Sec. III we present a multipole based tracking code that was developed for global systematic parameter scans that is validated against CST Studio Suite's particle-incell (PIC) solver (Appendix). In this section we also introduce the orthogonal multistaging concept and the optical matching of these stages. In Sec. IV, we exploit the multipole based tracking algorithm and study the beam dynamics of single and double structure chains towards a global optimization study as a function of focusing and particle amplitude as well as a range of energy gain per stage. In this section we also compare the beam dynamics under $\mathrm{THz}$ fields with single frequency with a finite frequency band showing that effects due to the bandwidth (radial variation of transverse voltages due to different frequency components) are canceled and hence exert no significant modification on beam emittance and energy spread. Finally, in Sec. V we conclude that dielectric lined rectangular waveguides can generate a longitudinal field mode which lacks a monopolar transverse voltage component for relativistic beams, preventing emittance growth during the interaction unlike more conventional structures. Furthermore, we demonstrate that by employing an orthogonal multistaging scheme, the transversely correlated slice energy spread introduced by the $\mathrm{THz}$ field can be corrected up to $82 \%$, alongside the transverse emittance, conserving the six-dimensional phase space for the studied regime of up to $0.5 \mathrm{GV} / \mathrm{m}$ accelerating fields. 


\section{MULTIPOLE ANALYSIS OF THE THZ FIELD}

A group of orthonormal modes identified as longitudinal section modes (LSM) with no magnetic $\left(H_{y}\right)$ and electric $\left(E_{y}\right)$ components normal to the interface, are supported in a rectangular waveguide by the introduction of two dielectric slabs to the top and bottom face of the structure (see the inset of Fig. 1). These modes are derived from Hertz potentials using the vacuum-dielectric boundary conditions as given in [25].

The most appropriate mode for interaction with a beam is the $\mathrm{LSM}_{11}$ mode which provides a nonzero longitudinal electric field $(0 \leq y \leq a)$ corresponding to the odd mode for $m=1$, and $n=1$, where $m$ and $n$ are the number of half wavelengths for the horizontal and vertical axis. In this section, the $\mathrm{LSM}_{11}$ mode is decomposed to its multipole components and the characteristics of each component, alongside their effect on the beam dynamics, are discussed. The form of the longitudinal component of $\mathrm{LSM}_{11}$, which is used for acceleration, is given by

$$
\begin{aligned}
E_{z}(x, y, z, \omega)= & A\left(-i k_{z}\right) k_{y}^{a} \sin \left[\frac{\pi}{w}\left(x+\frac{w}{2}\right)\right] \cos \left(k_{y}^{a} y\right) \\
& \times e^{i \omega_{c}^{z}-k z+\phi},
\end{aligned}
$$

where $k_{z}$ is the propagation constant inside the waveguide, $k_{y}^{a}$ is the wave number in the $y$ direction in vacuum, which also the axis where the dielectric-vacuum boundary is located, $A$ is the amplitude independent of the frequency and geometry of the structure, $\omega$ and $k$ are the angular frequency and the total propagation constant of the $\mathrm{THz}$ field. The constant $k_{y}^{a}$ can be derived from the dispersion relation

$k_{y}^{a} \sin \left[k_{y}^{b}(b-a)\right] \sin \left(k_{y}^{a} a\right)=\epsilon_{r} k_{y}^{a} \cos \left(k_{y}^{a} a\right) \cos \left[k_{y}^{b}(b-a)\right]$,

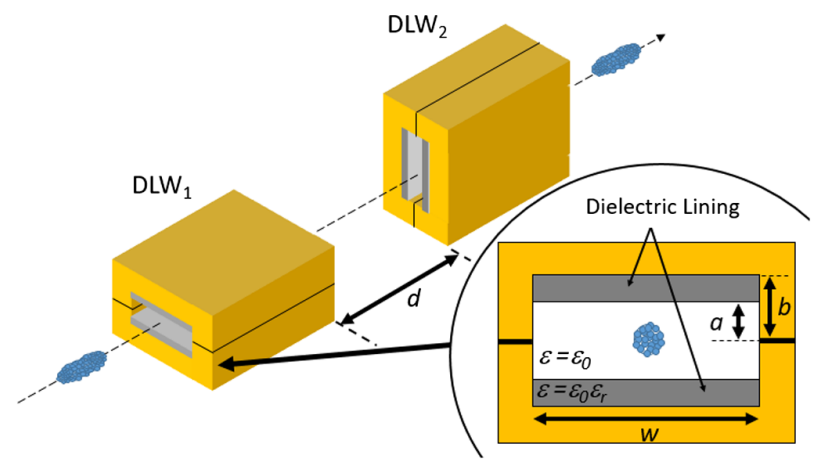

FIG. 1. Schematic diagram of the rectangular dielectric-lined waveguide (DLW) structure and the alternating orientation of two waveguides used for staged acceleration with separation $d$. The DLWs were designed for synchronism to relativistic electrons therefore, operating at $0.465 \mathrm{THz}$ with $a=250 \mu \mathrm{m}, b=300 \mu \mathrm{m}$ and $w=1.2 \mathrm{~mm}$. The permittivity of the vacuum and dielectric slab are denoted by $\epsilon_{0}$ and $\epsilon_{r}$, respectively. and according to $k_{y, m n}^{a}=\sqrt{k_{0}^{2}-\left(\frac{m \pi}{w}\right)^{2}-\beta_{m n}^{2}}$. The solutions of the dispersion relation are shown in Fig. 2 for a range of longitudinal propagation constants $\beta=k_{z}$. According to this, a single frequency $\mathrm{THz}$ field $(0.465 \mathrm{THz})$ synchronizes to a relativistic electron beam $\left(v_{p} \approx c\right)$ when $k_{0}=\beta=9746$ that results in an imaginary wave number in the $y$ direction, $k_{y}^{a}=i \pi / w$. Furthermore, assuming a single frequency and $v_{p} \approx c$, the phase relation $\left(e^{i \omega z / c-i k z}=1\right)$ is satisfied and the longitudinal field can be written independent of longitudinal position, $z$. The potential associated to the longitudinal component of $\mathrm{LSM}_{11}$ can be rearranged in polar coordinates to

$V_{z}(r, \theta)=-i A \beta L k_{y}^{a} \cos \left(\frac{\pi}{w} r \cos (\theta)\right) \cos \left[k_{y}^{a} r \sin (\theta)\right]$,

where $\theta$ is the azimuthal angle and $r$ is the radial position. Hence both the longitudinal field and potential have a strong transverse dependence. Consequently, we perform a multipole analysis up to the fourth order (higher orders were found to be negligible) in order to identify the individual components and the effects on phase space of the beam. We note that the Fourier expansion of the longitudinal field is

$V_{z}(r, \theta)=\frac{1}{2} a_{0}+\sum_{n=1}^{\infty} a_{n} \cos (n \theta)+\sum_{n=1}^{\infty} b_{n} \sin (n \theta)$.

In this expansion, the monopole component is $1 / 2 a_{0}$ and higher-order components are calculated up to the octupolar component $(n=4)$. The Fourier coefficients $a_{0}, a_{n}$ and $b_{n}$ are calculated using the integrals

$$
a_{0}=\frac{1}{\pi} \int_{-\pi}^{\pi} V_{z}(r, \theta) d \theta
$$

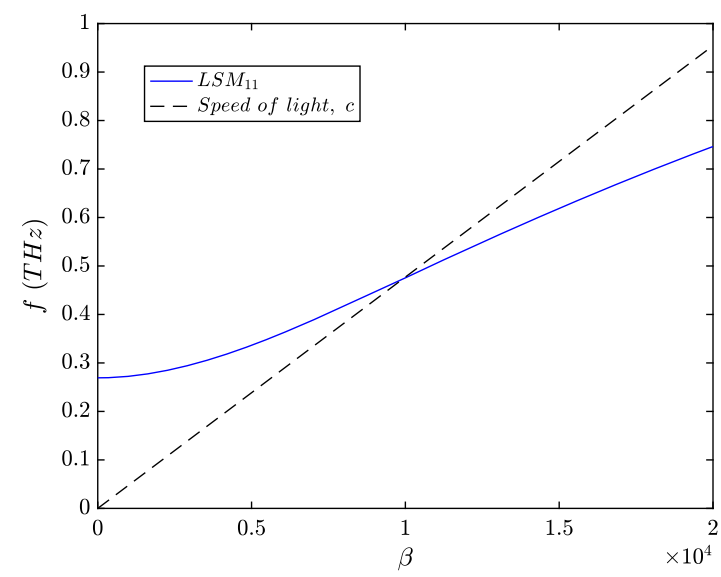

FIG. 2. Dispersion plot for $\mathrm{LSM}_{11}$ mode synchronous to a relativistic electron beam $\left(v_{p} \approx c\right)$. 


$$
\begin{aligned}
& a_{n}=\frac{1}{\pi} \int_{-\pi}^{\pi} V_{z}(r, \theta) \cos (n \theta) d \theta, \\
& b_{n}=\frac{1}{\pi} \int_{-\pi}^{\pi} V_{z}(r, \theta) \sin (n \theta) d \theta .
\end{aligned}
$$

We substitute $V_{z}(r, \theta)$ and Fourier components are numerically calculated up to the fourth order through

$$
\begin{array}{r}
a_{0}=\frac{\alpha}{\pi} \int_{-\pi}^{\pi} \cos \left(\frac{\pi}{w} r \cos \theta\right) \cos \left(k_{y}^{a} r \sin \theta\right) d \theta, \\
a_{n}=\frac{\alpha}{\pi} \int_{-\pi}^{\pi} \cos \left(\frac{\pi}{w} r \cos \theta\right) \cos \left(k_{y}^{a} r \sin \theta\right) \cos (n \theta) d \theta, \\
b_{n}=\frac{\alpha}{\pi} \int_{-\pi}^{\pi} \cos \left(\frac{\pi}{w} r \cos \theta\right) \cos \left(k_{y}^{a} r \sin \theta\right) \sin (n \theta) d \theta,
\end{array}
$$

where $\alpha=-i A \beta L k_{y}^{a}$ is the magnitude of the potential for practicality.

The multipole components of $E_{z}$ are calculated by normalizing each Fourier component of $V_{z}$ by the axial monopole term, $V_{0}$, for the frequency where the phase velocity is synchronous with a fully relativistic beam as seen in Fig. 3(a). We found that dipole and sextupole components, as well as higher-order components, above fourth order are negligible, leaving only a radially constant monopole, quadrupole and a weak octupole component of $E_{z}$ acting on the beam dynamics.

All multipole components under consideration for the longitudinal $\mathrm{THz}$ field have a strong radial dependence except from the radially constant monopole component. According to the Panofsky-Wenzel theorem,

$$
V_{\perp}=-\frac{i c}{\omega} \int_{0}^{L} d z \nabla_{\perp} E_{z}(z, z / c),
$$

the transverse gradient of the longitudinal multipole components induce transverse voltages shown in Fig. 3(b). These voltages are calculated across a longitudinal interaction distance $L$ and for a field magnitude of $100 \mathrm{MV} / \mathrm{m}$, a typical target value for DLW work to reach the limit of conventional accelerators [26].

One immediate result is that the lack of a transverse voltage due to the monopole component on a beam traveling synchronous to the $\mathrm{THz}$ field as $\nabla E_{z \text {,monopole }}=0$. This is a unique feature of dielectric waveguides and it limits the transverse emittance growth, in contrast to most conventional radio-frequency structures [27,28].

We have shown that for a rectangular geometry the $\mathrm{THz}$ field is dominated by the quadrupole component. This is in contrast to cylindrical waveguides, where only a monopole component exists and higher order components cancel due to symmetry. The characteristic transverse distribution of $E_{z}$, including quadrupole and octupole components, is presented in Fig. 4(a), where a saddle point occurs due

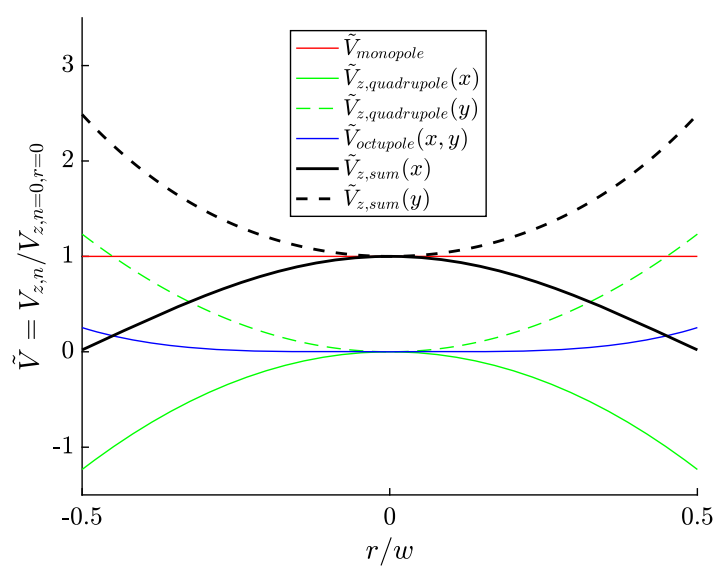

(a)

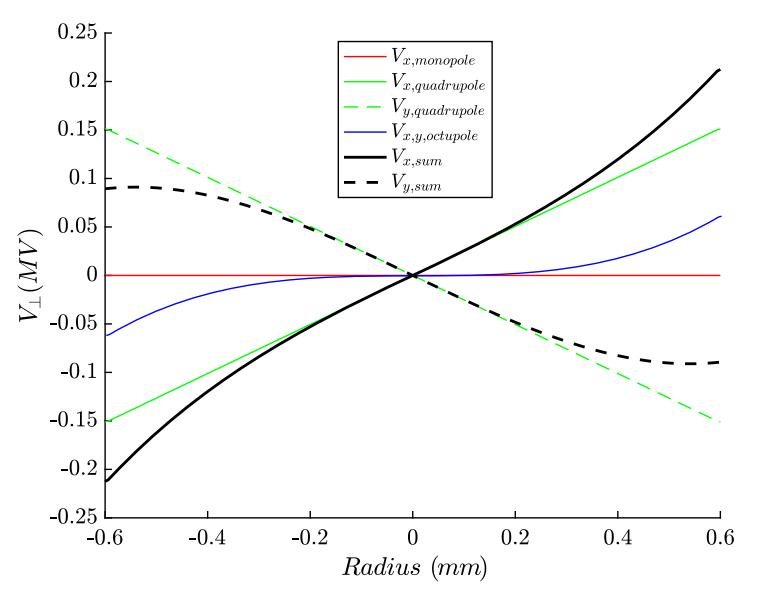

(b)

FIG. 3. Pure monopole, quadrupole and octupole components of the longitudinal potential and their sum in (a) for $0.465 \mathrm{THz}$ and the geometry given in Fig. 1. (b) The corresponding transverse voltage for quadrupole and octupole components according to the Panofsky-Wenzel theorem for an amplitude of $100 \mathrm{MV} / \mathrm{m}$. The radially constant longitudinal monopole field component yields a zero transverse monopolar voltage.

to the opposite sign of the quadrupole component on horizontal and vertical axes. An electron beam incident to a $\mathrm{THz}$ field with such a distribution with $100 \mu \mathrm{m}$ radius samples an rms field deviation of $7 \mathrm{MV} / \mathrm{m}$ for an on-axis $100 \mathrm{MV} / \mathrm{m}$ accelerating gradient. This introduces a correlated energy spread on the beam proportional to the beam radius and interaction length.

In order to reduce or completely remove the induced slice energy spread during the THz interaction, we studied a scheme employing a pair of structures orthogonal to each other (as shown in Fig. 1) counteracting the quadrupolar transverse distribution of the longitudinal field and compensating against the correlated energy spread on the beam [25]. Figure 4(b) presents the field distribution after a second identical waveguide that is rotated by $90^{\circ}$. As can be seen in the figure, the energy spread due to the quadrupole 


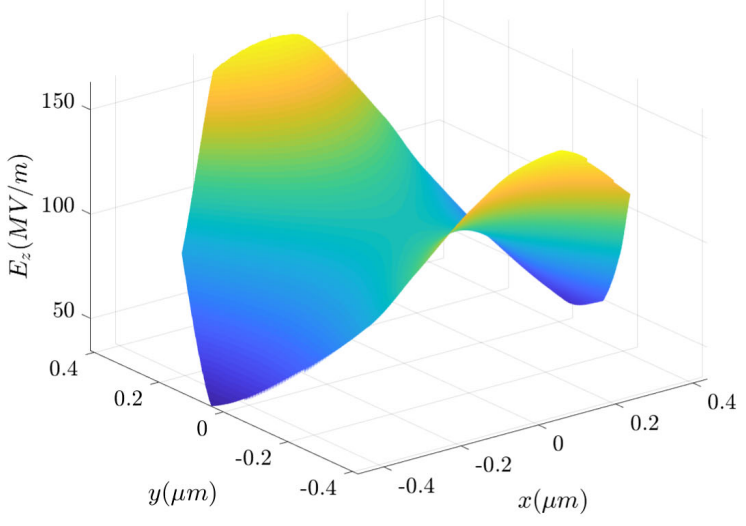

(a)

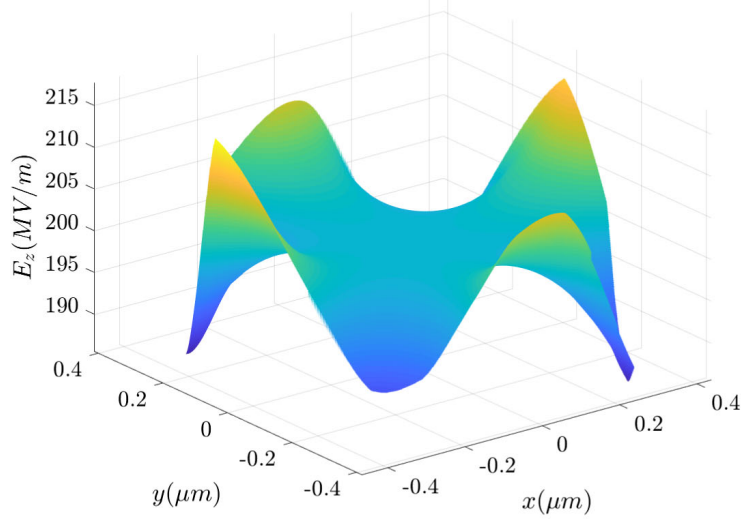

(b)

FIG. 4. (a) The rms beam energy as a function of transverse coordinates in a rectangular waveguide correlated with the transverse dependency of quadrupole component of $E_{z}$. Results are for a slice electron beam with $100 \mu \mathrm{m}$ radius resulting in a $7 \mathrm{MV} / \mathrm{m}$ rms field deviation. (b) The energy distribution for the same beam after propagating through the orthogonal multistage arrangement. In the latter, the correlated rms spread in the field is reduced down to $0.5 \mathrm{MV} / \mathrm{m}$.

component is corrected and a weak octupole component is the only source of correlated energy spread with a $0.5 \mathrm{MV} / \mathrm{m}$ deviation in the transverse plane.

This nonexistence of a monopolar component combined with the use of an orthogonal multistage arrangement opens up the ability to preserve both transverse emittance and energy spread. A rectangular waveguide is therefore an appealing candidate to fill the gap between high gradient novel schemes where beam quality is compromised and conventional schemes with high $6 \mathrm{D}$ brightness, albeit with low accelerating gradients.

\section{MULTIPOLE BASED PARTICLE TRACKING}

Following the multipole analysis of the $\mathrm{THz}$ field, an algorithm was developed to track the $6 \mathrm{D}$ phase space coordinates of particles under the impact of these individual components of $E_{z}$. This approach improves the computing efficiency by 2 orders of magnitude compared to particlein-cell solvers (such as PIC solver in CST Microwave Studio), and provides a tool for extensive systematic beam dynamics studies. According to the thin lens approach used, particles are tracked through multiple slices in the direction of movement where the longitudinal and transverse impact, or kicks, are calculated on particle momenta during the interaction with terahertz field. A linearly decreasing field amplitude was considered across the subsequent kicks, resembling a realistic amplitude profile during terahertz propagation due to finite bandwidth. This profile, presented in Fig. 5, is calculated using CST Particle Studio for a narrowband pulse with a central frequency of $0.465 \mathrm{THz}$ and a bandwidth of $100 \mathrm{GHz}$. This is done to accurately capture the pulse walk-off and phase slippage in a real THz field for the acceleration of a single on crest electron, and sufficient for our studies as we consider the THz pulse spectrum fixed and only wish to study the effect of the amplitude and electron bunch distribution. The particles are defined in 6D canonical phase space and matched to the optical functions at the point of creation.

During each kick, a change in total momentum is applied according to the Lorentz force as $\Delta p=e E_{z}\left(L_{\text {slice }} / c\right)$, where $L_{\text {slice }}$ is the length over which the kick is applied. The time dependence of the longitudinal electric field is given by

$$
\begin{aligned}
E_{z}(x, y, z)= & A\left(-i k_{z}\right) k_{y}^{a} \sin \left[\frac{\pi}{w}\left(x+\frac{w}{2}\right)\right] \\
& \times \cos \left(k_{y}^{a} y\right) \cos \left(\omega \frac{z}{v_{z}}+\phi\right),
\end{aligned}
$$

where $z$ and $v_{z}=\beta_{z} c$ are longitudinal position and velocity of each particle and $\phi$ is the arbitrary initial phase.

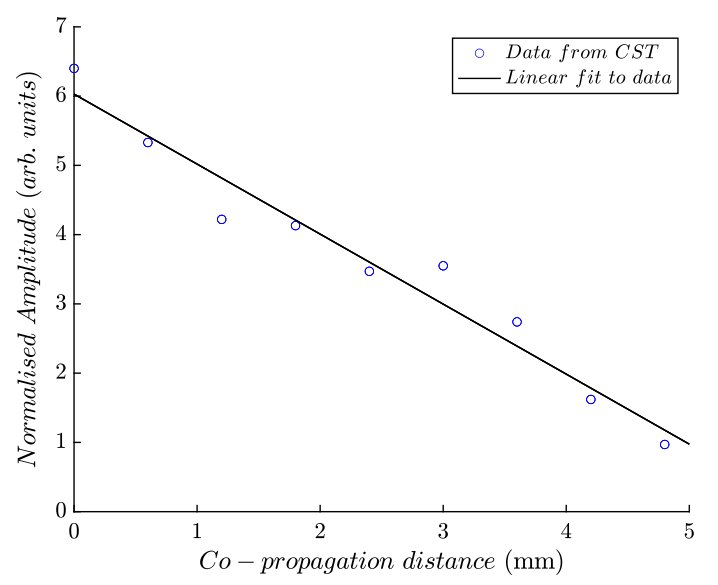

FIG. 5. The spatial profile of the THz field amplitude due to finite bandwidth as it copropagates synchronously with relativistic electrons. 


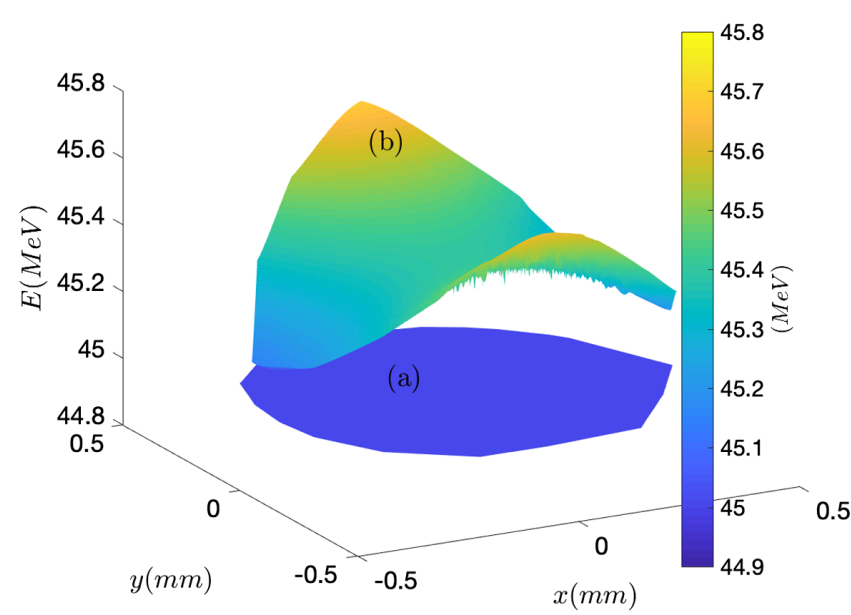

FIG. 6. (a) The flat initial energy surface around $45 \mathrm{MeV}$ without uncorrelated energy spread. (b) The transverse energy distribution after interaction with the $\mathrm{THz}$ field correlated with the dominant quadrupole component.

The particles also receive a transverse kick due to the transverse voltage, $V_{\perp}$, causing a change in the particle angles according to $\Delta x^{\prime}=e V_{\perp} / E(z)$, where $E(z)$ is the initial mean energy of the particle beam at the start of each slice. After each kick particles drift through the slice with final positions according to $x_{\text {final }}=x(z)+L_{\text {slice }} \Delta x^{\prime}$, where $x(z)$ is the particle position at the start of the slice and $\Delta x^{\prime}$ is the change in the particle angle. The total interaction length is divided into multiple sections of kick-drift pairs to provide accuracy.

For a beam interacting with the $\mathrm{THz}$ field dominated by the quadrupole component, the total energy spread consists of the initial uncorrelated energy spread, $\delta E_{0}$ and the correlation with $E_{z}$ as a function of time and transverse coordinates. For simplicity, a zero initial uncorrelated energy spread was defined for the initial distributions used for the studies presented here and time and radial correlation are referred to as longitudinal and transverse correlation. Figure 6(a) presents such a monoenergetic, Gaussian beam slice at $45 \mathrm{MeV}$ with $\sigma_{x, y, 0}=100 \mu \mathrm{m}$ radius and $\sigma_{z, 0}=50 \mathrm{fs}$ bunch length. These parameters reflect the design goal for CLARA, but the beam dynamics results will be valid for relativistic energies in general. After interaction with the $\mathrm{THz}$ field, the transverse energy distribution is modified by strong correlation to the quadrupole component of $E_{z}$ as shown in Fig. 6(b).

\section{A. Orthogonal multistaging}

The six-dimensional brightness is a key merit for beam quality which is achieved for beams with a minimal footprint in position-angle and energy-phase spaces as well as carrying high charge per bunch. The slice properties are particularly important for applications such as free electron lasers (FELs) operating at self-amplified spontaneous radiation (SASE) mode where the radiation source becomes slices within a bunch in the longitudinal axis themselves through a process called microbunching [29]. Therefore, a high 6D brightness is crucial to produce a narrow-band high brightness FEL light.

In Sec. II, we have demonstrated the strong dependence of transverse energy distribution to the quadrupole component of the $\mathrm{THz}$ accelerating field. This introduces a transversely correlated energy spread which is stronger for larger beam sizes, as the quadrupole component increases with radius. We now show that orthogonal multistaging counteracts the effects of transverse field distribution and preserves the slice properties in the six-dimensional phase space. In the following examples, we will demonstrate the scheme using two rectangular structures, rotated $90^{\circ}$ relative to each other and separated with an arbitrary drift length or a magnetic matching section.

After the THz interaction in the first structure, particles continue diverging towards the second structure with increasing beam radius due to nonzero rms beam angle. In the second stage, this leads to overcompensation of energy for particles located at larger radii due to the sum of quadrupole and octupole electric field components increasing nonlinearly with radius. This reduces the performance of correlated energy spread correction. However, to improve the correction efficiency a matching section can be introduced in between the stages to return each particle to their original transverse coordinates corresponding to those at the entrance of the first structure. Consequently, particles originally sampled the maxima of the quadrupoledominated transverse field distribution undergo the minima at the second structure with right counteracting transverse gradient and vice versa. This is the mechanism resulting in the cancellation of the correlated slice energy spread induced by the first stage. In this section, we will explore a point-to-point transformation of the particle coordinates by a pseudoinverse matching algorithm to achieve a complete cancellation of correlated slice energy spread. However, the transformation obtained from this initial solution is not necessarily physical and so we propose a hybrid method using the nonperiodic point-to-point transfer matrix to identify physical solutions close to the transformation (for $x, x^{\prime}$ ) suggested by pseudoinverse matching.

\section{Pseudo-inverse matching}

The transformation of the initial beam positions $\left(x_{1 n} \cdots x_{1 N}\right)$ and angles $\left(\theta_{1 n} \cdots \theta_{1 N}\right)$ of each particle via a transfer matrix, $R_{\mathrm{DLW}}$, is shown in Eq. (13),

$$
\left(\begin{array}{l}
x_{21} \cdots x_{2 N} \\
\theta_{21} \cdots \theta_{2 N}
\end{array}\right)=R_{D L W}\left(\begin{array}{c}
x_{11} \cdots x_{1 N} \\
\theta_{1.1} \cdots \theta_{1 N}
\end{array}\right)
$$

where $n=1,2,3, \ldots, N$ is the index of each particle and $N$ is the total number of particles. The transfer matrix through the first structure and the drift section separating the two structures is denoted as $R_{\mathrm{DLW}}$ that yields new particle 
positions $\left(x_{2 n} \cdots x_{2 N}\right)$ and angles $\left(\theta_{2 n} \cdots \theta_{2 N}\right)$ at the entrance of the second structure.

We use a Moore-Penrose pseudoinverse in order to derive the matrix, $R_{\mathrm{DLW}}$ for known initial and final particle positions and angles, representing the beam transformation from the entrance of the first structure to the end of the drift section. The transformation of position and angle of each particle constitutes a system of $N$ linear equations where the initial beam coordinates matrix is given as $X$ and the final beam coordinates matrix, $Y$. These matrices are related through the transfer matrix as in $Y=R_{\mathrm{DLW}} X$ and define a set of linear equations for positions and angles of each particle. The pseudoinverse operation can be used to find the least squares solution to a system of linear equations, i.e., the elements of the transfer matrix, $R_{\mathrm{DLW}}$.

For example, a 1 fs-long slice, focused at the entrance of the first DLW with an rms beam waist of $24 \mu \mathrm{m}$ and a rms divergence of 4 mrad was tracked across the two DLW structures, $\mathrm{DLW}_{1}$ and $\mathrm{DLW}_{2}$, separated by a short drift section of $10 \mathrm{~mm}$. The beam area expands by a factor of 6 at the entrance of $\mathrm{DLW}_{2}$ after drifting which causes a factor of 6 increase in the correlated energy spread sampled. This transverse energy correlation after the first and second DLWs are presented in Figs. 7(a) and 7(b), respectively. A matching section can reduce the beam size after the first DLW, ideally to its initial value at the entrance. Figure 7(c) shows a $99 \%$ reduction in rms slice energy spread by counteracting the transverse correlation with the quadrupole component of the longitudinal field when the pseudoinverse matching algorithm is implemented. After this correction, a residual transverse coupling to the weak octupole component becomes visible in the transverse energy profile.

For the same example, the evolution of beam envelope across the DLW structures is presented in Fig. 8(a) comparing the matched and unmatched cases. The corresponding slice energy spread evolution as a function of distance traveled is shown in Fig. 8(b) demonstrating a complete cancellation of the correlated slice energy spread in the second DLW when the beam is matched, and an increase, proportionate to the beam area, when the beam is left unmatched between the two DLWs.

The recovery efficiency of the slice energy spread using the orthogonal multistaging scheme depends on the beam size hence the type and strength of the multipole components sampled from the transverse field distribution. A systematic scan of energy spread as a function of rms beam radius through an orthogonal multistage is useful to understand the transverse correlation of beam energy with the longitudinal multipole components. The same beam in the previous example was tracked through an orthogonal multistage with no drift section in between the two stages

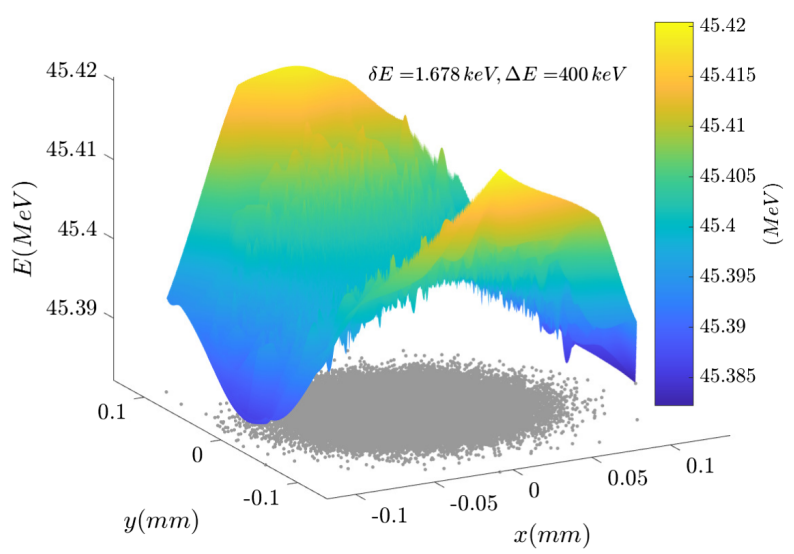

(a)

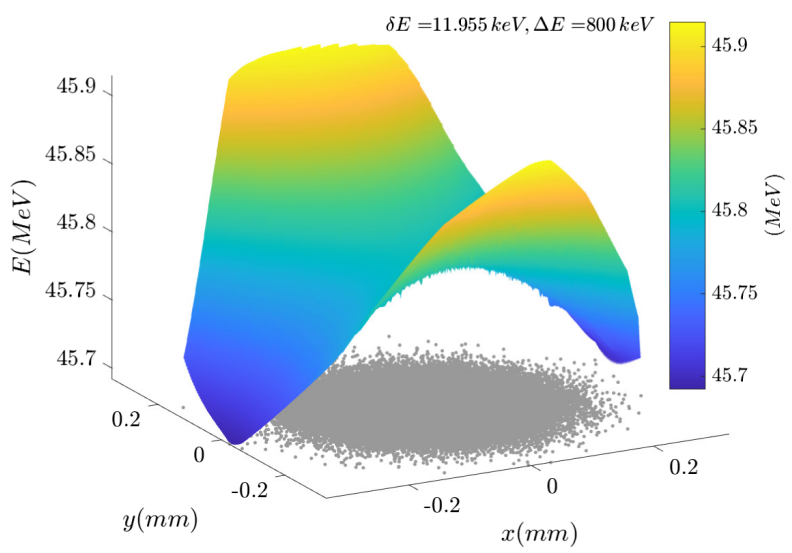

(b)

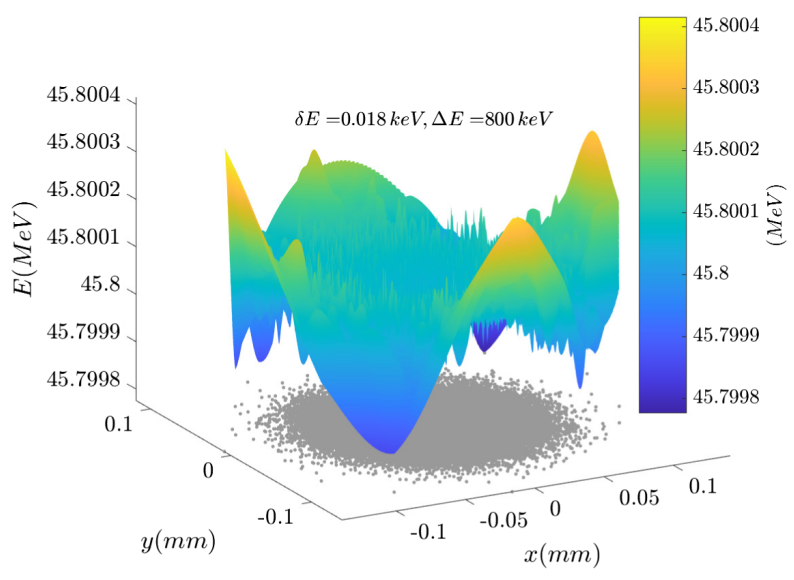

(c)

FIG. 7. Slice energy spread strongly correlated to the quadrupole component of $E_{Z}$ for an initially $45 \mathrm{MeV}$ beam. (a) Propagated through a single DLW, (b) two DLW stages separated with a $10 \mathrm{~mm}$ drift section without matching and (c) after the drift length is removed and matching algorithm employed. Results are for $400 \mathrm{keV}$ energy gain per stage. 


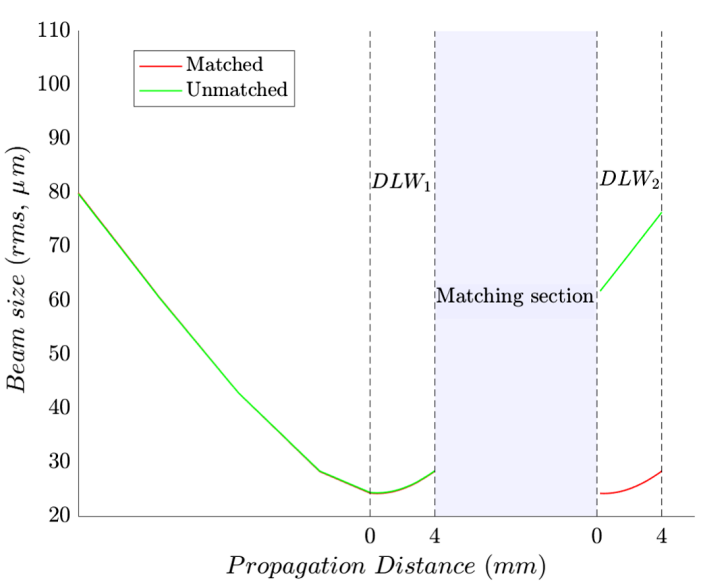

(a)

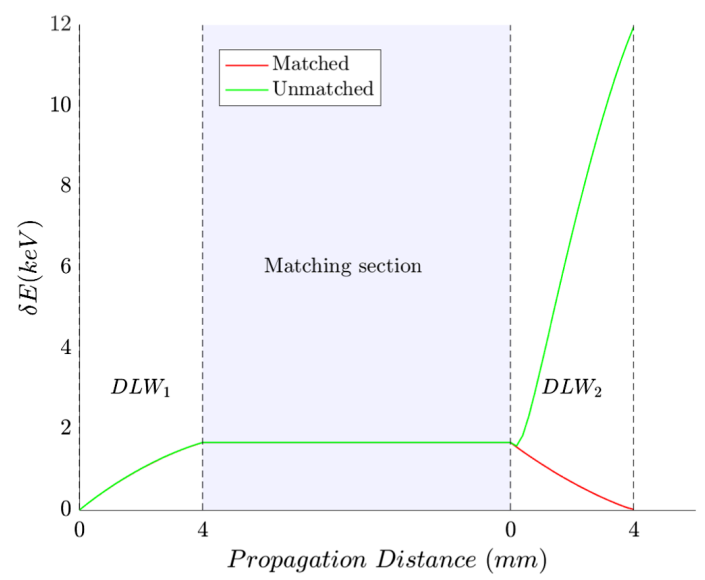

(b)

FIG. 8. (a) Beam envelope evolution through an orthogonal two-stage system with and without a pseudoinverse matching between stages. (b) The energy spread profile across a multistaged system. Entrance and exit of DLWs are marked with vertical dashed lines where the entrance of the first DLW is located at zero.

to monitor the behavior purely under the multipole fields free from additional increase in the beam radius in the drift section. Figure 9 demonstrates that the energy spread increases with radius as expected and that the slice energy spread induced by the first DLW can be counteracted by the second orthogonal DLW. The amount of correction achieved slightly decreases (99\% to $92 \%$ for a beam radius of 30 to $\sim 100 \mu \mathrm{m}$ ) with increasing radius as more of the octupole component is sampled which cannot be corrected with the orthogonal multistaging scheme.

\section{Correction under nonperiodic transformation}

In the previous section we showed that the compensation of the transversely correlated energy spread significantly improves by implementing a matching section in between the two DLWs. As mentioned previously, $R_{M}$ represents a

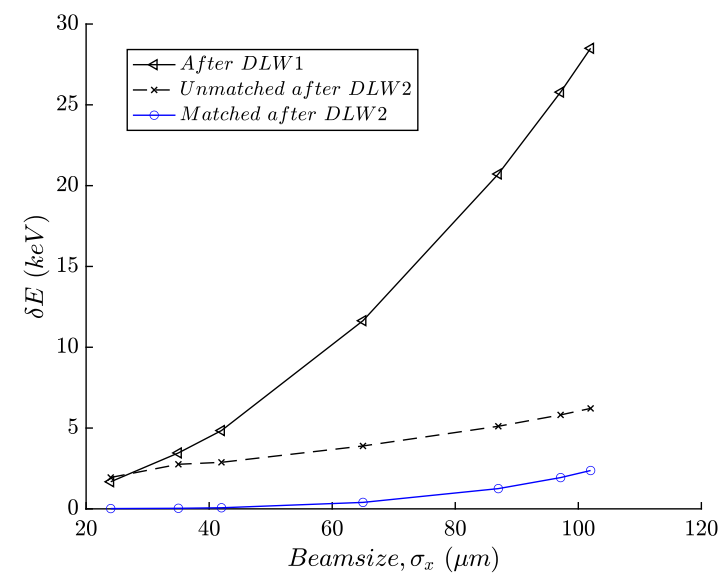

FIG. 9. The transversely correlated energy spread as a function of incoming rms beam size at the entrance of the first DLW. Results are presented at the exit of $\mathrm{DLW}_{1}$ and at the exit of $\mathrm{DLW}_{2}$ for a matched and an unmatched beam. No drift section is introduced between the stages to monitor the pure response of staging without the increase in beam size due to a drift section.

$2 \times 2$ matrix allowing a point-to-point matching of particle positions and angles at the entrance points of the first and second DLW for complete compensation of the correlated energy spread. A physical lattice that provides this transformation can be explored by performing a least-square fitting to the matrix elements of various quadrupole magnet arrangements.

On the other hand, the feasibility of $R_{M}$ can also be tested through the investigation into transformation characteristics of Twiss parameters between the stages using the definition of well-known nonperiodic transfer matrix, $R_{i j}$, giving the transformation of the beam parameters between any two arbitrary points, $s_{0}$ and $s_{1}$ in the lattice without requiring the periodicity condition for Twiss parameters.

In order to construct the nonperiodic transfer matrix, the Twiss parameters are extracted from the particle distribution at the exit of $\mathrm{DLW}_{1}$ and entrance of $\mathrm{DLW}_{2}$ using the definition of the beam matrix, $\Sigma$, in Eq. (14),

$\Sigma=\left(\begin{array}{ll}\sigma_{11} & \sigma_{12} \\ \sigma_{21} & \sigma_{22}\end{array}\right)=\left(\begin{array}{cc}\left\langle x^{2}\right\rangle & \left\langle x x^{\prime}\right\rangle \\ \left\langle x^{\prime} x\right\rangle & \left\langle x^{\prime 2}\right\rangle\end{array}\right)=\left(\begin{array}{cc}\beta & -\alpha \\ -\alpha & \gamma\end{array}\right) \epsilon$,

i.e., $\alpha=-\left\langle x x^{\prime}\right\rangle / \epsilon$ and $\beta=\left\langle x^{2}\right\rangle / \epsilon$ where quantities between \langle\rangle denote rms values and $\gamma$ is a Twiss parameter. Figure 10 presents the elements of the nonperiodic matrix $M$ as a function of phase advance. Each set of $\mathrm{R}_{i j}$ corresponding to a given phase advance represents a physically allowed transformation. The transfer matrix elements predicted by a pseudoinverse matching algorithm are indicated with horizontal dashed lines in the figure.

According to Fig. 10, although there is a solution including $R_{11}, R_{21}$ and $R_{22}$ around a phase advance of $2 \pi$, there is no single phase advance satisfying the values given by pseudoinverse matching for all four matrix 

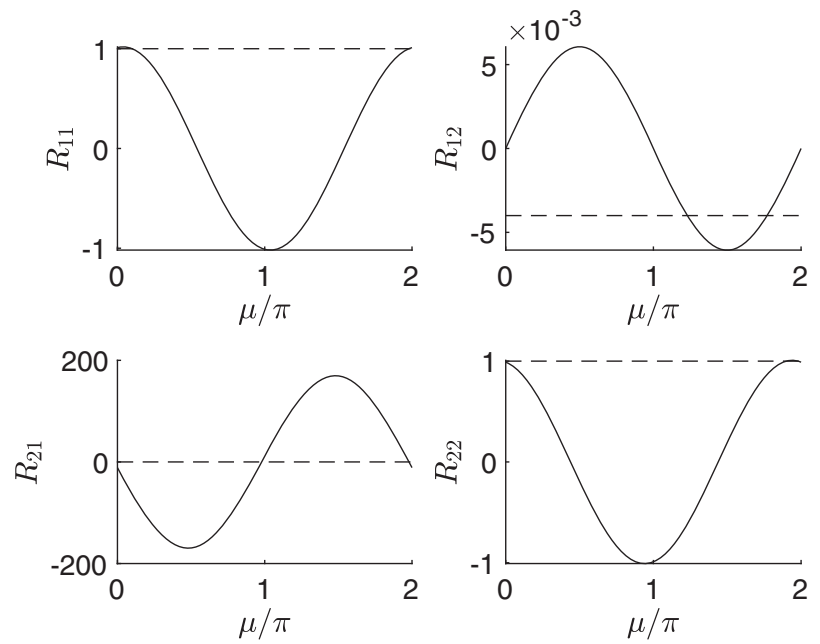

FIG. 10. Elements of nonperiodic transfer matrix over a range of phase advances for a beam with an rms radius of $24 \mu \mathrm{m}$ and an

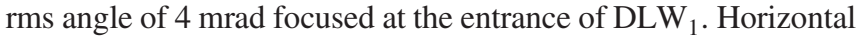
dashed lines indicate the values predicted by the pseudoinverse matching.

elements. We shall see that $R_{12}$ is the most critical matrix element in terms of energy spread recovery (Fig. 12). Therefore, we determined sets of $R_{i j}$ at phase advances around the reference nominal $R_{12}$ suggested by the pseudoinverse algorithm.

These solutions from Fig. 10 are presented in Table I and compared in terms of the resulting energy spread at the end of $\mathrm{DLW}_{2}$. An optimum matrix, minimizing the resulting energy spread, can be interpolated using these discrete solutions. Figure 11 presents the interpolation between these points to determine the $R_{12}$ value yielding the maximum energy spread correction with a minimum final energy spread of $0.31 \mathrm{keV}$ (also included in Table I). The beam envelopes and energy spread profile corresponding to each solution specified in the table are presented in Fig. 12 in comparison to the perfectly matched (PME) solution using a pseudoinverse algorithm. This figure also justifies our interest in $R_{12}$ for energy spread reduction.

To sum up the results so far, the analysis of nonperiodic matrix reveals that matrix elements $\mathrm{R}_{i j}$ suggested by the

TABLE I. The solutions of nonperiodic transfer matrix for the matching section at given phase advances and resulting final energy spread values for each solution. The residual energy spread is minimized for the solution at $\mu=1.816$.

\begin{tabular}{lllcll}
\hline \hline$\mu(\pi)$ & $\mathrm{R}_{11}$ & \multicolumn{1}{c}{$\mathrm{R}_{12}$} & $\mathrm{R}_{21}$ & $\mathrm{R}_{22}$ & $\Delta \mathrm{E} / \mathrm{E}(\mathrm{keV})$ \\
\hline 1.98 & 1.001 & -0.0004 & -0.0001 & 0.98 & 1.7 \\
1.883 & 0.898 & -0.0022 & 50.25 & 0.99 & 0.75 \\
1.834 & 0.81 & -0.003 & 74.83 & 0.96 & 0.35 \\
1.816 & 0.77 & -0.0033 & 83.3 & 0.94 & 0.31 \\
1.803 & 0.75 & -0.0035 & 89.19 & 0.92 & 0.33 \\
1.77 & 0.672 & -0.004 & 103.7 & 0.8711 & 0.5422 \\
\hline \hline
\end{tabular}

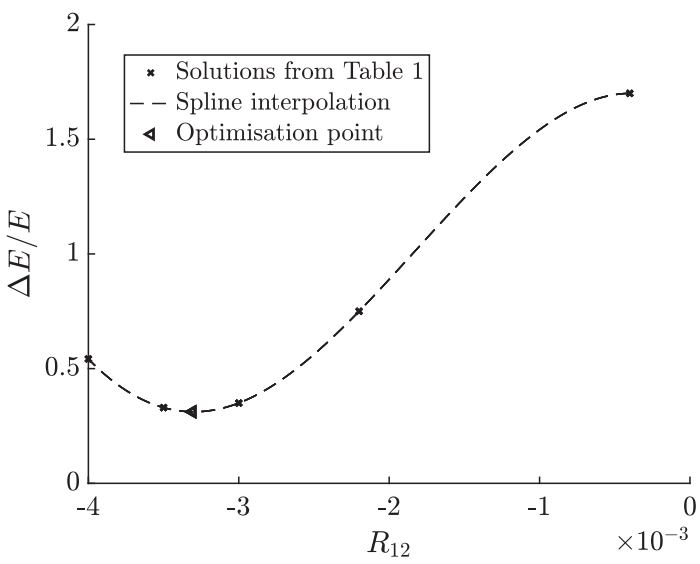

FIG. 11. The energy spread as a function of the most sensitive transfer matrix element $\mathrm{R}_{12}$. Data points from Table $\mathrm{I}$ are interpolated to determine the $R_{12}$ value yielding the minimum energy spread of $0.31 \mathrm{keV}$ at the end of $\mathrm{DLW}_{2}$.

pseudoinverse matching might not always be satisfied simultaneously. Instead, one should optimize a final matching matrix finding a trade-off between $R_{12}$ and $R_{21}$ which is also consistent with the nonperiodic transverse matrix of the lattice. A matching lattice performing point to point transformation resulting in maximum compensation of the induced correlated energy spread is highly sensitive to $R_{12}$ value, hence, this quantity is the figure of merit of our optimization. Nevertheless, with a matching section after $\mathrm{DLW}_{1}$ which focuses the beam into $\mathrm{DLW}_{2}$, all solutions presented provide a final slice energy spread smaller than the value observed $(12 \mathrm{keV})$ under unmatched conditions. Our studies indicate that a realistic matching lattice satisfying the solutions suggested by pseudoinverse matching refined by the nonperiodic matrix approach is possible via strong focusing with a quadrupole triplet lattice $<1 \mathrm{~m}$. These results are presented in next subsection.

\section{A realistic matching lattice}

We explored the feasibility of realistic focusing lattices consisting of quadrupole magnet arrangements for their performance of providing the desired matching quality. The doublet arrangements proved insufficient to generate the negative $R_{12}$ element, therefore we focused our studies on quadrupole triplet arrangements. This is done by simultaneous least square minimization of matrix elements resulted from pseudoinverse operation with corrections from the nonperiodic matrix and a quadrupole triplet with variable quadrupole strengths for given quadrupole and drift lengths between the quadrupoles.

Table II presents examples of triplets including a design with no drift sections in between, and subsequent variations labeled as $Q \#$ for quadrupoles; $D_{1} \#, D_{2} \#$ and $D_{3} \#$ for the drift sections in between the quadrupoles and after the triplet, respectively, where \# gives the length of that particular element in millimeters. The table also presents 


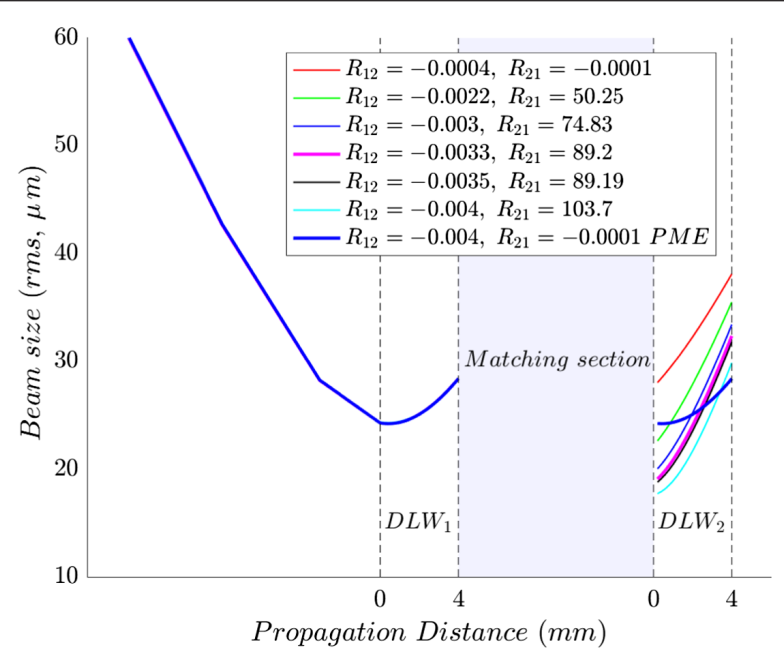

(a)

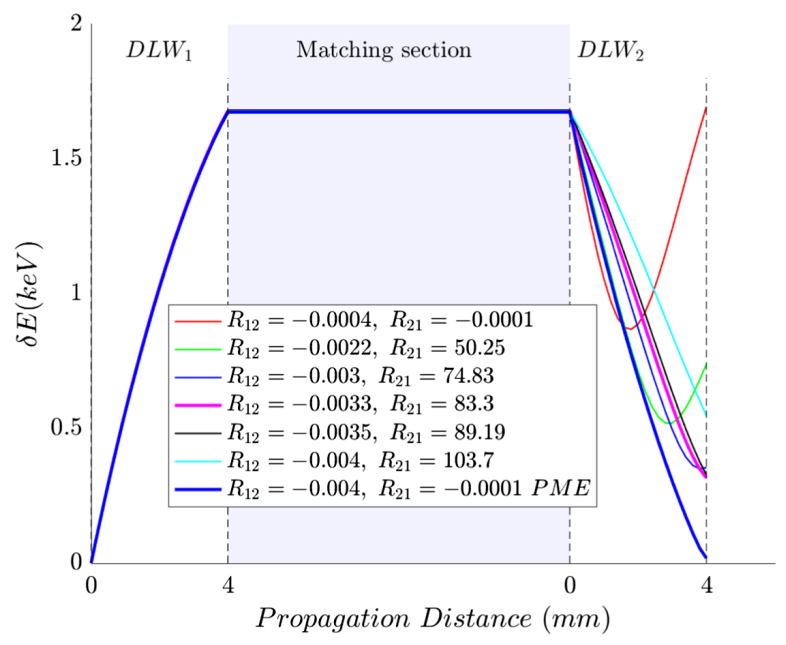

(b)

FIG. 12. (a) The beam envelope focused at the entrance of $\mathrm{DLW}_{1}$ and across $\mathrm{DLW}_{2}$ after the matching section resulting from each solution presented in Table I. (b) The increase of energy spread due to the correlation with the longitudinal quadrupole component of the $\mathrm{THz}$ field in $\mathrm{DLW}_{1}$ and recovery of this correlated energy spread in $\mathrm{DLW}_{2}$ corresponding to a different solution for the matching section.
TABLE III. The elements of the transfer matrices corresponding to different triplet designs.

\begin{tabular}{lcrrr}
\hline \hline Design & $R_{11}$ & \multicolumn{1}{c}{$R_{12}$} & \multicolumn{1}{c}{$R_{21}$} & \multicolumn{1}{c}{$R_{22}$} \\
\hline No drift & 0.6897 & -0.0033 & 13.1928 & 0.9039 \\
Q100D 10 & 0.7845 & -0.0033 & 51.5138 & 0.9738 \\
Q50D 10 & 0.7629 & -0.0033 & 97.2900 & 0.9003 \\
Q100D $150 D_{2} 150$ & 0.8118 & -0.0035 & 129.0208 & 0.9870 \\
Q100D $200 D_{2} 200$ & 0.9157 & -0.0037 & 139.9490 & 1.1135 \\
Q100D $200 D_{2} 200 D_{3} 200$ & 0.7461 & -0.0033 & 46.8733 & 0.9202 \\
\hline \hline
\end{tabular}

the total length required by each matching section design, as well as the quadrupole strengths given by $g(\mathrm{~T} / \mathrm{m})$. The quadrupole tip radius required to provide each strength is given as $r(\mathrm{~mm})$ and presented in the table for each quadrupole in the triplet. During the least square minimization, all quadrupoles were set to the same length while their strengths were varied. For the calculation of $g$, we considered that the flux density in the steel will increase as one goes further out from the pole tip, and if it gets towards $\sim 2 \mathrm{~T}$, the pole can get highly saturated [30]. That is often taken as a sign that the fields are at the high end of feasibly achievable. Therefore, the magnetic flux density at the pole tip was kept fixed and equal to $1 \mathrm{~T}$ during these calculations. Furthermore, the current required to power a quadrupole goes as $N I=g r^{2} / 2 \mu_{0}$, where $\mathrm{N}$ is the number of turns per coil, I is the current and $\mu_{0}$ is the magnetic permeability of free space. Therefore, the size of the aperture is a compromise between the current value and the clearance required for the beam pipe.

The transfer matrices for each matching section design are included in Table III. Different designs were integrated into the tracking code by means of their transfer matrices and final energy spread values were evaluated from these tracking results (Table II). According to this, $Q 50 D_{3} 10$ design produces a transfer matrix closest to the one concluded in Table I which also achieves the lowest energy spread as well as the shortest lattice $16 \mathrm{~cm}$ in length. However, the matching section design requires some small apertures of only a few millimeters which would have practical drawbacks. On the other side, $Q 100 D_{3} 10$ design

TABLE II. The specifications of quadrupole triplet arrangements with different lattice lengths and resulting energy spread of the multistage.

\begin{tabular}{lcccc}
\hline \hline Design & Matching section length $(\mathrm{cm})$ & $\mathrm{g}(\mathrm{T} / \mathrm{m})$ & $\mathrm{r}(\mathrm{mm})$ & $\Delta E(\mathrm{keV})$ \\
\hline No drift & 30 & $20.4 / 111.5 / 7.3$ & $50 / 9 / 138$ & 0.92 \\
Q100D 10 & 31 & $75.3 / 0.5 / 66.2$ & $13.3 / 201 / 15.1$ & 0.54 \\
Q50 10 & 10 & $310.2 /-0.18 / 219.2$ & $3.2 / 567 / 4.6$ & 0.46 \\
Q100D $150 D_{2} 150$ & 40 & $48.8 /-1.8 / 50.2$ & $20 / 55.8 / 20$ & 0.49 \\
Q100D $200 D_{2} 200$ & 50 & $46.5 /-3.7 / 47.9$ & $21.5 / 268 / 21$ & 0.86 \\
Q100D $200 D_{2} 200 D_{3} 200$ & 65 & $61 / 1.2 / 10.5$ & $16.4 / 807 / 95.4$ & 0.5 \\
\hline \hline
\end{tabular}


provides an energy spread of $<1 \%$ of $800 \mathrm{keV}$ total energy gain. This design calls for a $31 \mathrm{~cm}$ long matching section.

Moreover, the performance of a realizable matching section also depends on the level of space charge forces acting upon the beam. The beam envelope under the space charge forces, ignoring the external focusing and acceleration, can be given as

$$
\sigma^{\prime \prime}-\frac{\kappa_{s}}{\sigma \beta^{3} \gamma^{3}}-\frac{\epsilon_{n}^{2}}{\sigma^{3} \beta^{2} \gamma^{2}}=0
$$

where $\sigma$ is the rms beam size, $\kappa_{s}$ is the beam perveance, $\beta$ and $\gamma$ are normalized beam velocity and energy and $\epsilon_{n}$ is the normalized beam emittance. In this equation, the last two terms represent the defocusing due to the space charge force and outward pressure due to beam emittance, respectively. Therefore the ratio of these two terms, $\epsilon_{n}^{2} \beta \gamma / \sigma^{2} \kappa_{s}$, is an indicator of balance between the space charge and emittance for a given beam. The initial experiments in CLARA [7] were performed using a 2 ps bunch with a charge of $60 \mathrm{pC}$ at $35 \mathrm{MeV}$. According to this the ratio between normalized emittance and space charge defocusing is $\sim 12$ for a typical $1 \mathrm{~mm}$ mrad emittance and $100 \mu \mathrm{m} \mathrm{rms} \mathrm{beam} \mathrm{size.} \mathrm{This} \mathrm{result} \mathrm{deems} \mathrm{any} \mathrm{of} \mathrm{the}$ matching performance degradation due to space charge effect negligible for our future tests and for this paper. Moreover, the results reported here regarding short bunches such as 1 and $50 \mathrm{fs}$ represent the slice properties rather than projected characteristics of a longer bunch.

Finally, when focused at the structure entrance, a CLARA-like beam with rms transverse size of $100 \mu \mathrm{m}$ and an rms divergence of $4.1 \mathrm{mrad}$, within the aperture limitations given in Fig. 1, can propagate $80 \mathrm{~mm}$ before it has contact with the vertical boundary of the structure. This, in principle, allows an active acceleration length of $80 \mathrm{~mm}$, if synchronization could be achieved over that length. We could hence cascade a series of acceleration sections each $80 \mathrm{~mm}$ long separated by matching sections of $31 \mathrm{~cm}$, i.e., over a total propagation length of $<0.4 \mathrm{~m}$, with $20 \%$ of the linac length providing acceleration.

\section{GLOBAL BEAM CHARACTERISTICS FOR SINGLE AND MULTISTAGE DLW}

Due to the constant monopolar component of longitudinal THz field, both slice and projected (when beam is focused at the entrance of $\mathrm{DLW}_{1}$ ) transverse beam emittances are preserved. We shall now investigate the impact of beam envelope characteristics on the transverse beam emittance and energy spread for a single DLW structure and a system of two structures located on orthogonal axes. This is done by using beam distributions at constant geometric emittance but with varying $\alpha$ and $\beta$ to represent a single electron source equipped with quadrupoles controlling the final focusing. Moreover, the investigation is done for varying bunch lengths of 1 fs representing the behavior of a single slice and 50 and 500 fs to observe the projected properties at an energy gain of $400 \mathrm{keV}$ per stage.

\section{A. Optimization of Courant-Snyder parameters}

A beam with parameters given in Table IV is injected into the DLW stages with zero initial uncorrelated energy spread as the scheme is designed to correct only correlated energy spread induced by the $\mathrm{THz}$ field. In order to ensure the statistical significance of rms calculations $10^{5}$ particles are used in tracking and space charge defocusing is not taken into account in the simulations. The behavior of a single slice is investigated as a function of $\alpha$, representing different focusing conditions resulting in different rms beam sizes at the entrance of the DLWs. Figure 13(a) demonstrates that the amount of correlated slice energy spread gained by the beam after the interaction within $\mathrm{DLW}_{1}(\delta E)$ is minimized down to $1.7 \mathrm{keV}$ when the beam is focused at the entrance of the first structure which corresponds to $\alpha=40$. Furthermore, in the same figure, the correlated spread acquired in a single stage can then be reduced by $82 \%$ after the interaction with a subsequent orthogonal stage $\mathrm{DLW}_{2}$.

As bunch length increases, the longitudinal correlation due to the time varying nature of the $\mathrm{THz}$ field becomes prominent. For example, the 50 fs case in 13(b) demonstrates that, for optimal focusing, the energy spread doubles from 6 to $12 \mathrm{keV}$ after the second structure. This is due to longitudinal correlation taking over the transverse correlation with increasing bunch length which cannot be removed with the orthogonal staging scheme. For the 50 fs case, for larger beam sizes in under- and overfocused regions, energy spread can still be recovered up to $70 \%$ due to dominant transverse coupling with the quadrupole component. As for the $500 \mathrm{fs}$ case the beam already spans through one fourth of a $\mathrm{THz}$ wavelength where the total energy spread is dominated by the longitudinal correlation in the case of a negligible uncorrelated energy spread. Consequently, the energy spread doubles due to longitudinal correlation for a significantly longer bunch.

In Sec. II, we showed that, for a rectangular waveguide, there is no transverse voltage acting on the beam due to the monopole component, contrary to symmetric structures where it exists and causes emittance growth. Furthermore, the transverse voltage induced by the quadrupole component has a linear radial dependence hence no slice emittance growth is induced as a result of the interaction with

TABLE IV. The characteristics of the electron beam used in tracking studies.

\begin{tabular}{lc}
\hline Geometric emittance, $\epsilon_{x, n}(\mathrm{~mm}$ mrad $)$ & 0.01 \\
Initial beam energy, $\mathrm{E}(\mathrm{MeV})$ & 45 \\
Number of tracked particles & $10^{5}$ \\
rms beam radius, $\sigma_{x}(\mu \mathrm{m})$ & 100 \\
rms beam angle, $\sigma_{x^{\prime}}(\mathrm{mrad})$ & 4 \\
\hline \hline
\end{tabular}




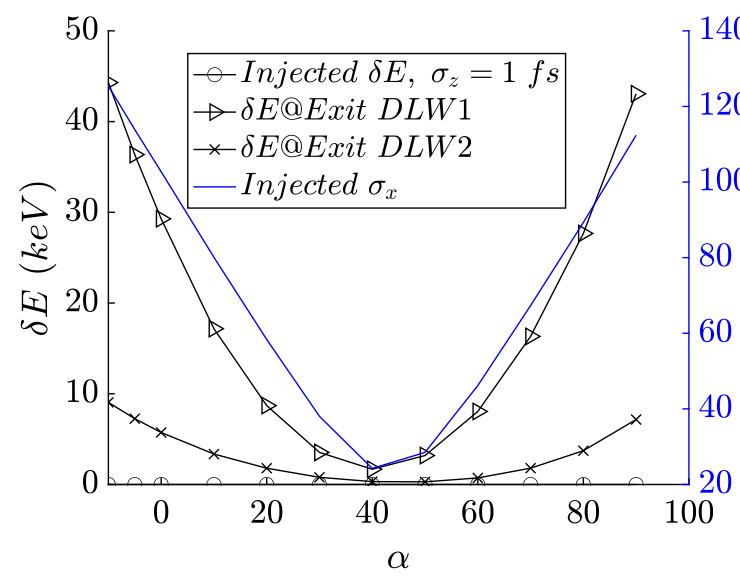

(a)

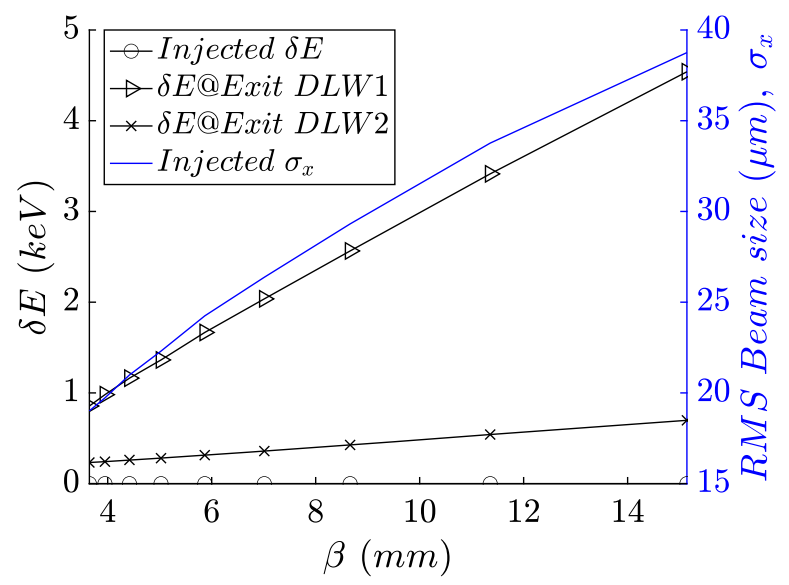

(c)

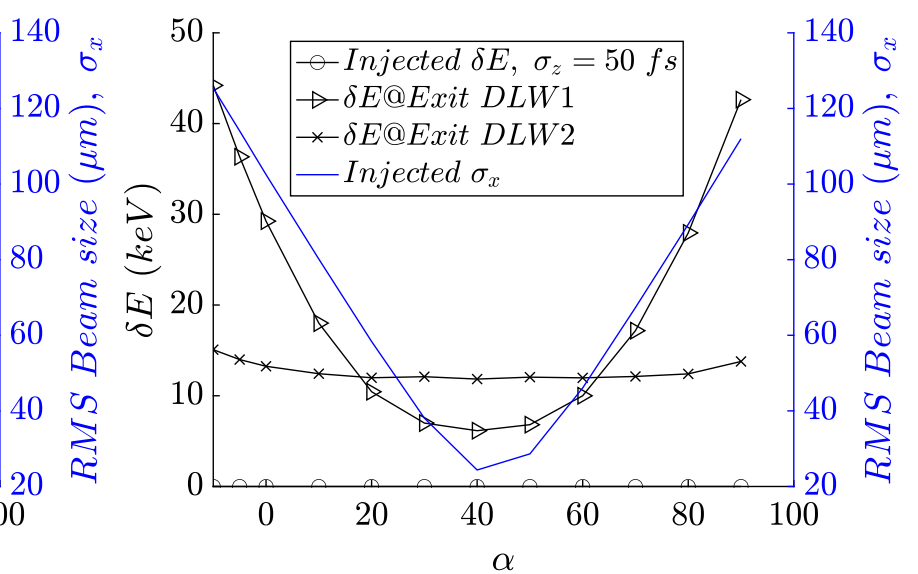

(b)

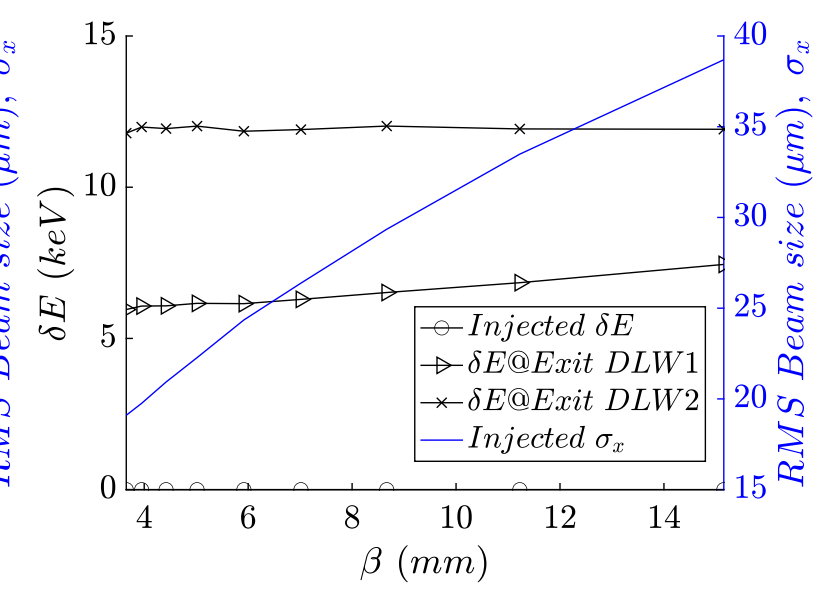

(d)

FIG. 13. Evolution of energy spread as a function of (a) and (b) focusing hence $\alpha$, and (c) and (d) $\beta$ for an initial emittance of $0.1 \mathrm{~mm}$ mrad and $400 \mathrm{keV}$ energy gain per DLW. The vertical columns (a) and (c) present results for rms bunch lengths of 1 and 50 fs, respectively (reading left to right for the bunch lengths). The results are generated under the optimized physical matching matrix described in Sec. III A.

the THz field. This is shown in 14(a) where an initial slice geometric emittance is constant across the stages, within statistical errors $(0.3 \%)$ for the same initial injection emittance and at a range of $\alpha$ values. However, each slice in a significantly longer bunch is impacted by a different amount of transverse kick due to the time dependency of the $\mathrm{THz}$ transverse voltage increasing the projected rms divergence, and hence projected emittance. This can be compensated by appropriate focusing as shown in Fig. 14(b) for a $500 \mathrm{fs}$ bunch. To sum up, both slice emittance and energy spread are preserved when an incident beam is focused at the entrance of an orthogonal double stage.

The correlated energy spread increases linearly with $\beta$ for a monotonically increasing beam size and constant geometric emittance. The behavior as a function of $\beta$ is presented in Figs. 13(c) and 13(d). The slice energy spread increases with the $\beta$ function hence the beam size as a larger section of the quadrupole field is covered by the beam. The cancellation of correlated energy spread via orthogonal staging reduced with increasing beam size. This is due to the inclusion of the nonlinear octupole component for larger radii. From 13(d) one can deduce that the recovery is limited to the amount of longitudinally correlated energy spread which is about $12 \mathrm{keV}$ for a 50 fs bunch for the optimum focusing. Similarly, for longer bunches of $500 \mathrm{fs}$, the total energy spread is completely dominated by the longitudinal correlation and it continues to increase across stages.

\section{B. Energy scaling}

The feasibility of using DLWs at higher energies is essential for the realization of the scheme for compact applications. Consequently, we investigated the performance of single and orthogonal-double stage up to an experimentally viable maximum energy gain of $2 \mathrm{MeV}$ per stage. Figure 15 shows this for a range of energy gains from $400 \mathrm{keV}$ to $2 \mathrm{MeV}$ per stage for slice (a) and projected (b) properties. 


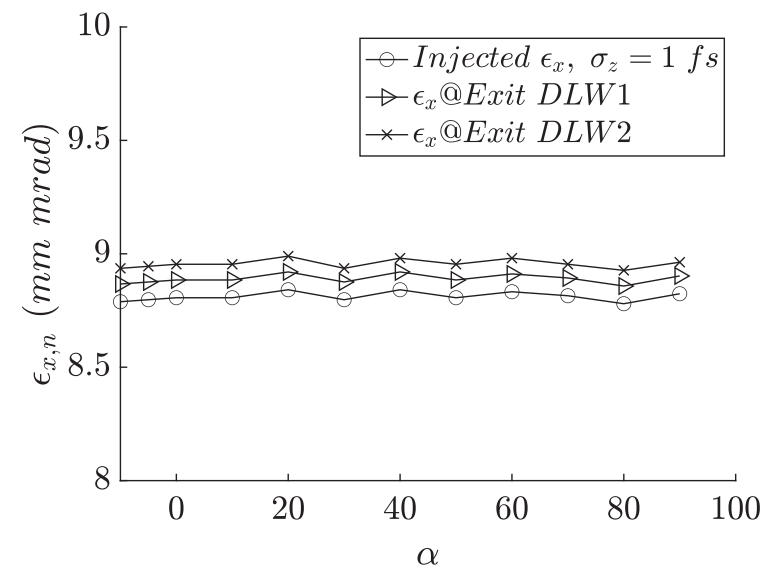

(a)

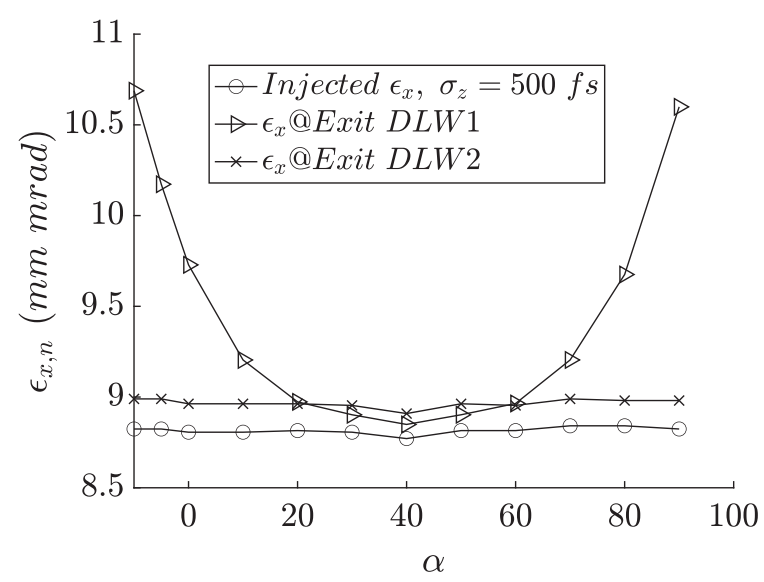

(b)

FIG. 14. Evolution of transverse beam emittance for (a) a slice ( $1 \mathrm{fs}$ ) and (b) a $50 \mathrm{fs}$ long bunch as a function of focusing conditions imposed while the beam is incident at the first structure entrance.

For a single DLW stage, the initial energy spread, $\delta E_{0}$, increases linearly with the energy gained after $\mathrm{DLW}_{1}$ while initial emittance at injection, $\epsilon_{0}$, stays constant provided that the beam is focused at the structure entrance. Figures 15(a) and 15(b) demonstrate the emittance preservation within the given range of energy gain for 1 and $50 \mathrm{fs}$ long bunches representing slice and projected characteristics, respectively. For the same focusing conditions, the increase in slice energy spread in a single structure can be corrected using orthogonal multistaging by about $82 \%$, whereas, the correction in the projected energy spread in (b) is shown to be limited by the longitudinal correlation for each energy level.

\section{The effect of bandwidth on beam quality}

The effect of the bandwidth of the THz field used for multipole-based tracking is taken into account by the variation in accelerating field with distance across the

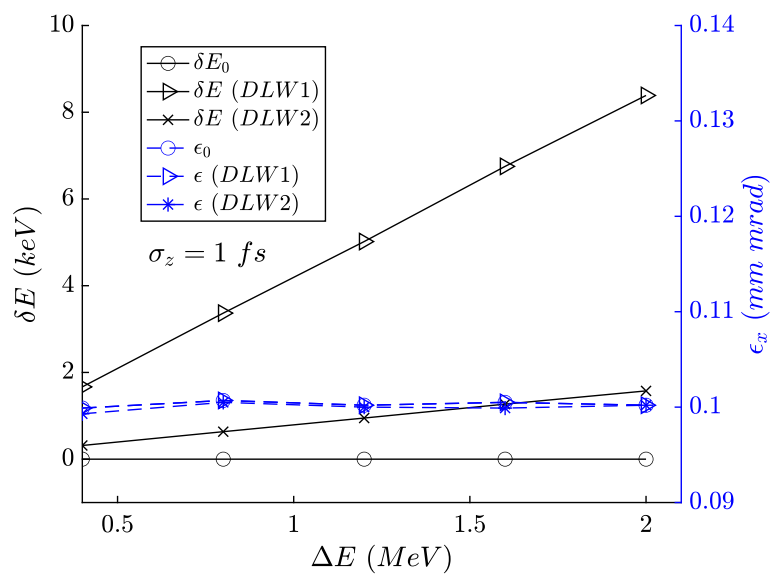

(a)

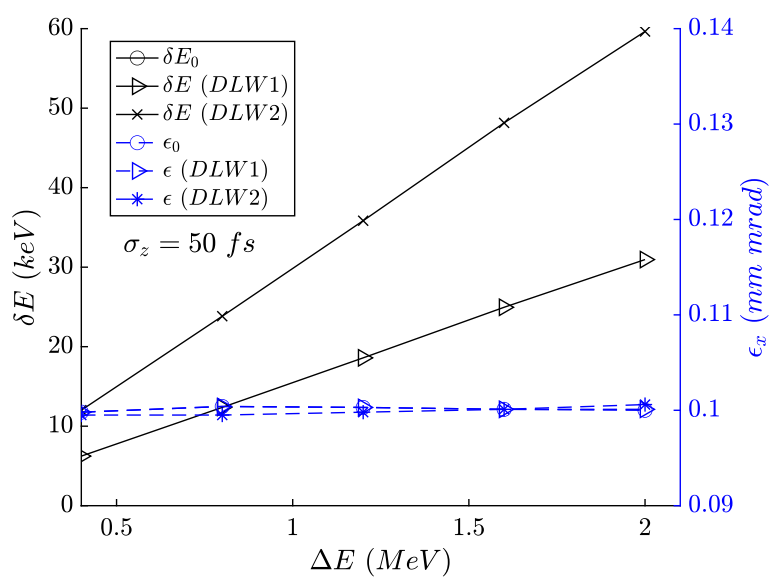

(b)

FIG. 15. Scaling of single-stage and multistage performance with energy gain per stage. (a) Slice energy spread and emittance, (b) projected energy spread and emittance for a $50 \mathrm{fs}$ beam $(\alpha=40) . \delta E_{0}$ and $\epsilon_{0}$ are initial energy spread and emittance at injection.

interaction length. However, we do not include the fact that the multipole components will vary with frequency and considered a single frequency $\mathrm{THz}$ field that is synchronous to a relativistic electron beam at $45 \mathrm{MeV}$ initial total energy. In reality, for different frequencies within a bandwidth, the monopole component will regain a radial dependency with opposite signs above and below the synchronous frequency where the total effect will mostly cancel which otherwise might cause emittance growth. Therefore, in order to explore the residual effects, we investigated the interaction of the beam with a broadband field where the central frequency $0.465 \mathrm{THz}$ is accompanied by two sidebands providing a finite bandwidth of $\pm 25 \mathrm{GHz}$ (standard deviation) each carrying $20 \%$ of the total amplitude.

Figure 16(a) presents the transverse voltage associated with the monopole component of the longitudinal $\mathrm{THz}$ field, for synchronous frequency only, either of the sidebands and for the total group that is a sum of all three 


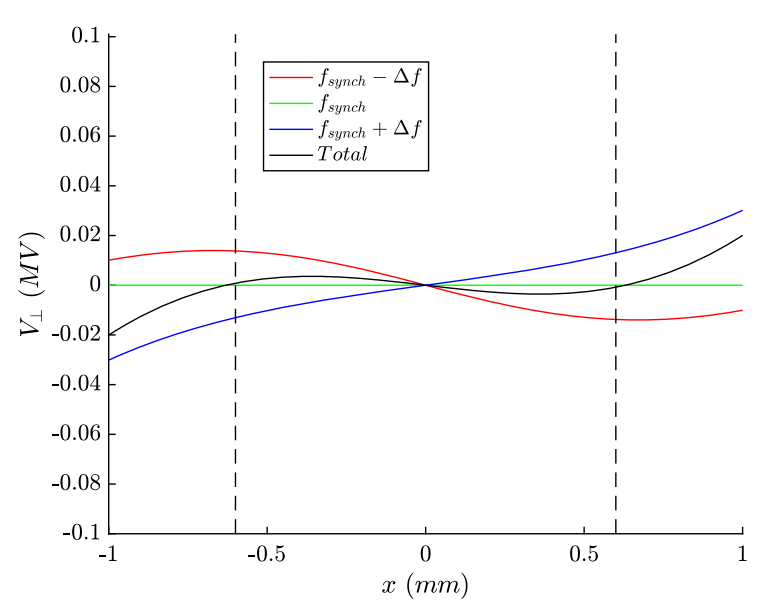

(a)

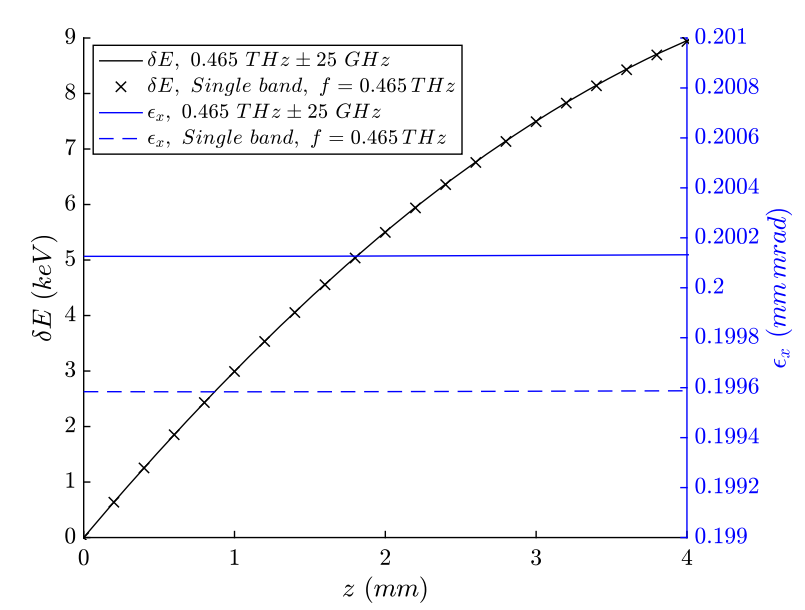

(b)

FIG. 16. (a) Transverse voltage regarding the longitudinal monopole component of the $\mathrm{THz}$ field for synchronous frequency, sidebands and their sum. (b) Effect of the total transverse voltage due to bandwidth on energy spread and emittance in a single structure. A beam with a geometric emittance of $0.2 \mathrm{~mm}$ mrad was tracked for both single frequency and broadband cases for an energy gain of $400 \mathrm{keV}$.

frequencies. As expected, the sum of the voltages from all contributing frequencies cancel out at the region of interest between $\pm 0.6 \mathrm{~mm}$, leaving a fractional transverse voltage. The impact of this residual voltage is shown in 16(b) where there is a $0.2 \%$ emittance growth for the broadband case in comparison to the single frequency, while no difference in energy spread is observed.

\section{CONCLUSIONS AND DISCUSSIONS}

The six-dimensional brightness of the particle beam is a key figure of merit for many applications of accelerators from light sources to particle colliders. However, minimizing both transverse and longitudinal phase space of a beam still remains challenging for emerging novel techniques.
In this paper, we demonstrated that a dielectric lined rectangular waveguide can preserve both emittance and energy spread, simultaneously, using an orthogonal multistaging scheme with implementation of appropriate multistaging coupling. We have demonstrated the concept of correlated energy spread correction via an orthogonal multistaging of dielectric lined rectangular waveguide structures.

In this study, the longitudinal multipole component of a $\mathrm{THz}$ field induced in a single structure was decomposed and the resulting transverse voltages were studied. It was shown that, for a $\mathrm{THz}$ field synchronous with electrons, a rectangular DLW, unlike conventional rf structures, lacks a monopole component of the transverse voltage, which is commonly a large contribution to the emittance growth of the beam during acceleration. Therefore, we have achieved the preservation of slice properties in the six-dimensional phase space both correcting for the induced correlated energy spread up to $82 \%$ while preserving the injected transverse emittance.

In order to study the interaction of a relativistic beam with the decomposed multipole components of the $\mathrm{THz}$ field and associated transverse voltages, a multipole based tracking code was developed and benchmarked against CST Studio Suites's PIC solver. This improved the computing speed significantly allowing global characterization of beam dynamics under $\mathrm{THz}$ fields.

Finally, we have studied the effect of a finite bandwidth $\mathrm{THz}$ field similar to that expected from real $\mathrm{THz}$ sources by demonstrating that the transverse voltage due to two sidebands around the central frequency mostly cancel, leaving a residual emittance growth of only $0.2 \%$ (within the statistical error of $0.3 \%$ ). The energy spread correlated to $\mathrm{THz}$ field also remains identical to the single frequency case.

\section{ACKNOWLEDGMENTS}

This work was supported by the United Kingdom Science and Technology Facilities Council (Grant No. ST/ P002056/1).

\section{APPENDIX: VALIDATION OF THE TRACKING CODE WITH SINGLE-STAGE}

CST Studio Suite includes a particle-in-cell solver allowing particles to be tracked through the fields generated in a defined waveguide structure excited by an initial THz pulse. A dielectric structure with a coupling section is modeled for these studies as shown in Fig. 17(a). The distribution of the $\mathrm{LSM}_{11}$ mode in the $y-z$ plane is presented in 17(b).

In the figure, the accelerating $\mathrm{LSM}_{11}$ mode occurs with the accelerating (blue) and decelerating (yellow) polarity as the field enters the waveguide followed by a transient mode excited in the coupler. The $\mathrm{LSM}_{11}$ mode is excited by a $0.465 \mathrm{THz}$ pulse with a bandwidth of $100 \mathrm{GHz}$ as shown in Fig. 18. The structure is excited by both real and imaginary 


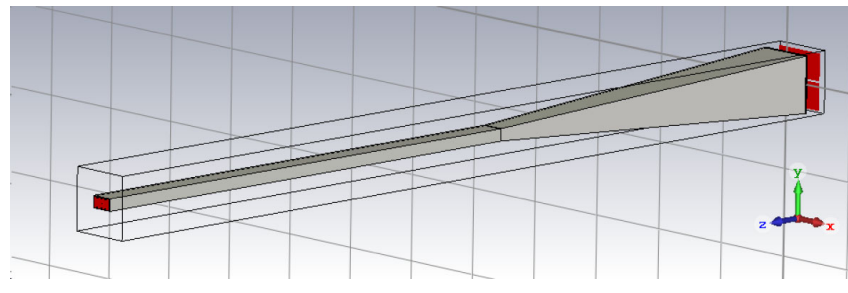

(a)

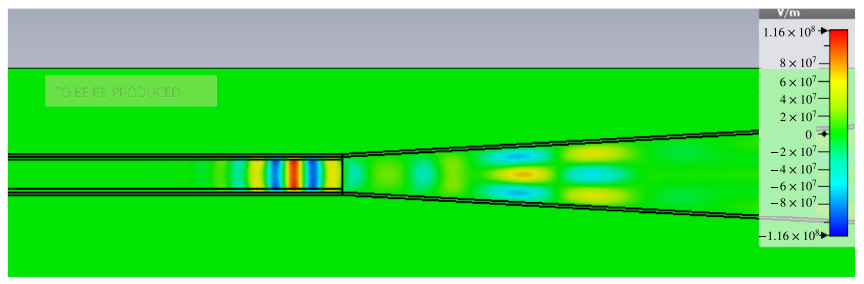

(b)

FIG. 17. (a) The $30 \mathrm{~mm}$ long rectangular structure with the $23 \mathrm{~mm}$ coupler section used in a CST benchmarking study. (b) Longitudinal electric field excited in the structure. The beam travels from right to left.

parts of the excitation field via two ports in CST to match the field profile of a Gaussian mode launched in free space via a short gap from the horn of the THz structure. A transverse cross section of the accelerating region regarding this induced $\mathrm{LSM}_{11}$ is presented in Fig. 19.

For benchmarking, a six-dimensional elliptical, 25 fs long beam slice at an energy of $45 \mathrm{MeV}$ with a radius of $10 \mu \mathrm{m}$ was externally generated with zero initial uncorrelated energy spread. This reference distribution was imported into CST and tracked through a $30 \mathrm{~mm}$ rectangular dielectric lined waveguide structure following a $23 \mathrm{~mm}$ coupling section using CST PIC solver. In this scenario, the incident beam drifts through the coupler and forms a waist at the entrance of the structure (focused at that location) until it intersects the $\mathrm{THz}$ field downstream the waveguide. We synchronized the electrons and the $\mathrm{THz}$ field by optimizing the emission timing of the two independently before they copropagate across the interaction length. In multipole based tracking, this was implemented as a $25.2 \mathrm{~mm}$ drift section identical to the CST PIC model.

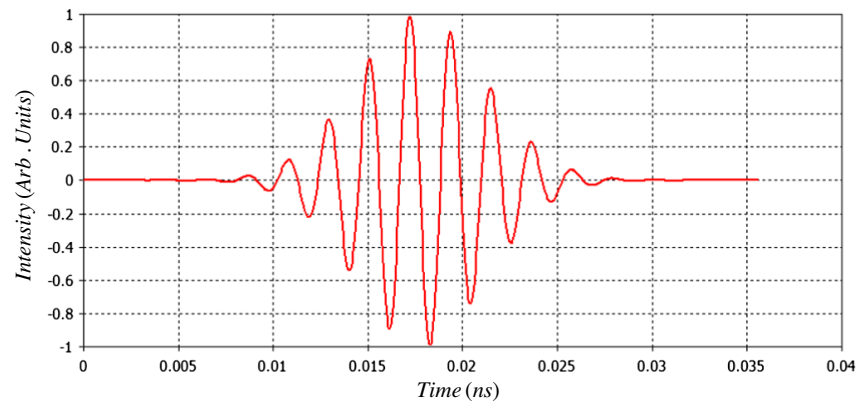

FIG. 18. Temporal profile of $0.465 \mathrm{THz}$ excitation signal with a bandwidth of $100 \mathrm{GHz}$.

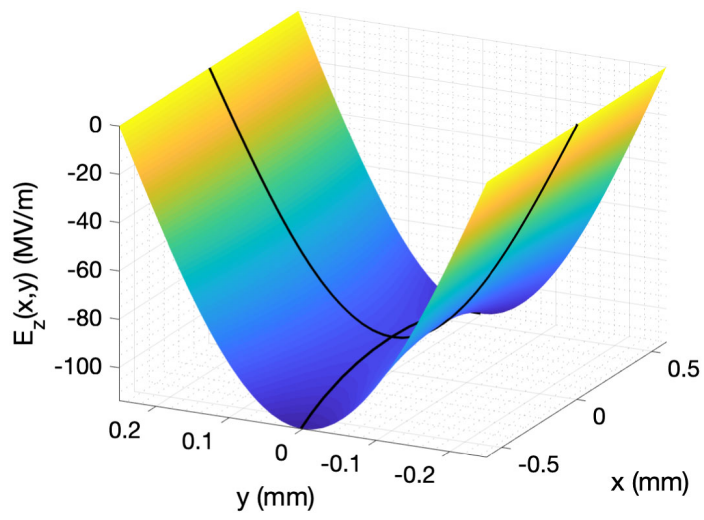

FIG. 19. A transverse central cross section of an accelerating phase of $E_{z}$ in CST that is dominated by the quadrupole component. The saddle point is denoted with intercepting axial lines.

After this drift section, the reference particle distribution was tracked under the fields introduced in Sec. II.

As previously mentioned, a THz field is synchronized to the electron beam providing that the phase velocity at the operating frequency $f_{0}$ is equal to the electron velocity, $v_{e}$. An electron beam will copropagate indefinitely with a monochromatic $\mathrm{THz}$ field. However, in reality the field has a finite bandwidth, $\Delta f$, and electrons will fall out of phase with frequencies other than $f_{0}$ as $v_{p} \neq v_{e}$. An interacting bandwidth is defined ensuring positive interaction between the electron and the field under the condition that within $\Delta f$ the phase slippage is less than $\pm \pi / 2$. The interacting bandwidth is given as in Eq. (A1) [31],

$$
\Delta f=\frac{1}{2 \pi} v_{e}\left(\beta\left(\omega_{2}\right)-\beta\left(\omega_{1}\right)+\frac{\pi}{L_{\mathrm{int}}}\right)
$$

where $\beta\left(\omega_{1}\right)$ and $\beta\left(\omega_{2}\right)$ are the wave numbers regarding the frequencies, $\omega_{1}$ and $\omega_{2}$, corresponding to bandwidth limits at $\pm \pi / 2$. Accordingly, the structure under study is designed at a length $L_{\text {int }}$ for a narrow-band $\mathrm{THz}$ pulse $(100 \mathrm{THz})$ [31]. In addition, at $45 \mathrm{MeV}$, electrons are ultrarelativistic and travel with the $99.99 \%$ of the speed of light whereas the group velocity for the $\mathrm{THz}$ pulse is about $60 \%$ of the speed of light. After including this group velocity walk-off, a final interaction length of about $4 \mathrm{~mm}$ is achieved.

The transverse energy distribution and phase space distribution from multipole-based tracking are compared to results from CST PIC solver in Figs. 20(a) and 20(b), respectively, for the identical initial beam distribution. The typical transverse energy distribution with a saddle point can be observed from both approaches with similar energy gain and energy spread values within $1 \%-2 \%$. The phase of the field in the tracking model was shifted by $\phi=\pi / 20$ to match the exact phase captured between the electrons and the THz field in the CST model. The benchmarking studies are performed for a 25 fs bunch in order to span over a few 


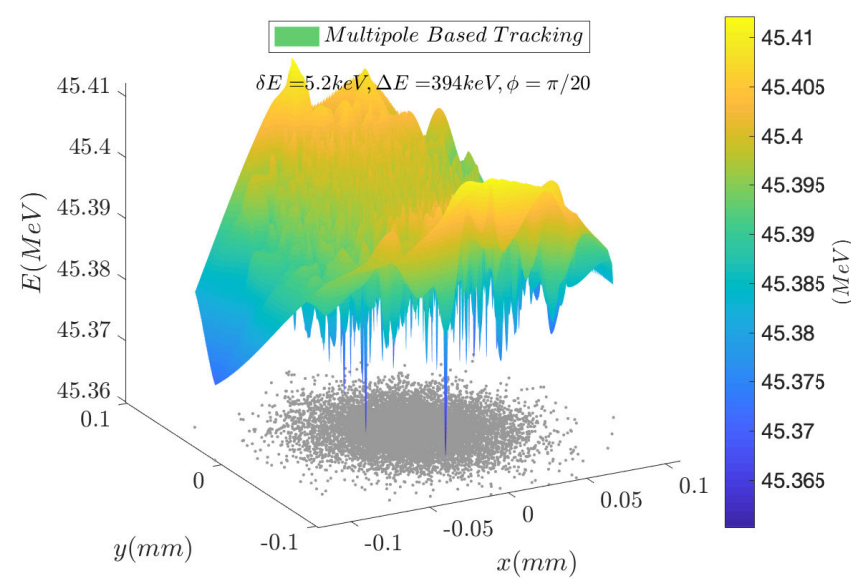

(a)

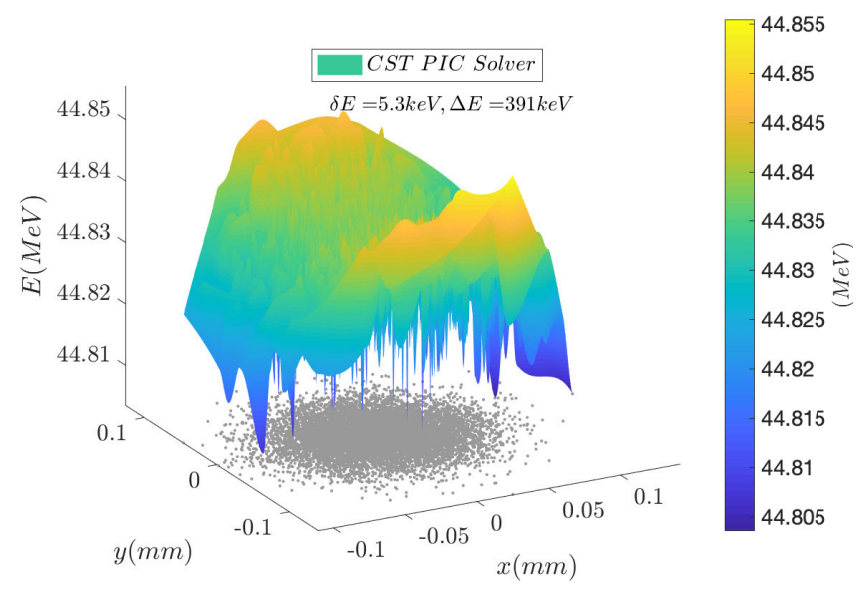

(b)

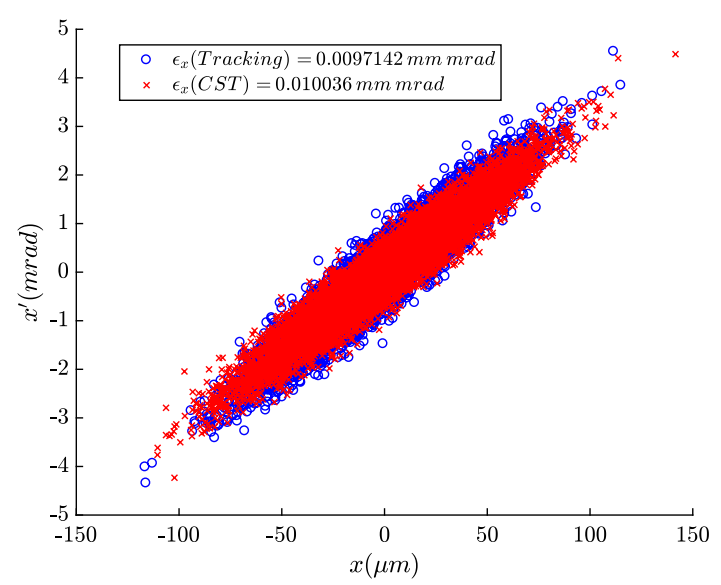

(c)

FIG. 20. The beam energy distribution in the $x-y$ plane after $4 \mathrm{~mm}$ copropagation with the accelerating polarity of the $\mathrm{THz}$ field (a) using multipole based tracking and (b) as a result of the CST PIC solver. The particle distributions with rms radius of $10 \mu \mathrm{m}$ and slice length of $25 \mathrm{fs}$ in the transverse plane are denoted with gray dots and the initial rms total energy is $45 \mathrm{MeV}$ for both cases. The CST PIC solver presents only the kinetic energy. The transverse phase space distributions are presented in (c). time steps in CST that is defined by the stability criterion, whereas, our multipole based tracking studies on beam characterization after interaction with $\mathrm{THz}$ will focus on the slice properties hence are performed for a slice of $1 \mathrm{fs}$ electron bunch. The emittance values from tracking and PIC simulations agree well within 3\%-8\% including 3\% statistical error induced by the sample size $\left(10^{5}\right.$ particles $)$ practically achievable by the CST PIC solver. The multipole based tracking code runs less than $6 \%$ of the computing time required by the CST PIC solver opening avenues for wide range parameter scans which will be reported in Sec. IV.

[1] Y. Li, G. Xia, K. Lotov, A. Sosedkin, and Y. Zhao, Highquality positrons from a multiproton bunch driven hollow plasma wakefield accelerator, Plasma Phys. Controlled Fusion 61, 025012 (2019).

[2] O. Mete, M. Labiche, G. Xia, and K. Hanahoe, GEANT4 simulations for beam emittance in a linear collider based on plasma wakefield acceleration, Phys. Plasmas 22, 083101 (2015).

[3] B. D. O'Shea, G. Andonian, S. K. Barber, K. L. Fitzmorris, S. Hakimi, J. Harrison, P. Hoang, M. Hogan, B. Naranjo, O. B. Williams, V. Yakimenko, and J. Rosenzweig, Observation of acceleration and deceleration in gigaelectronvolt-per-meter gradient dielectric wakefield accelerators, Nat. Commun. 7, 12763 (2016).

[4] M. A. K. Othman, J. Picard, S. Schaub, V. A. Dolgashev, S. M. Lewis, J. Neilson, A. Haase, S. Jawla, B. Spataro, R. J. Temkin, S. Tantawi, and E. A. Nanni, Experimental demonstration of externally driven millimeter-wave particle accelerator structure, Appl. Phys. Lett. 117, 073502 (2020).

[5] E. A. Nanni, V. A. Dolgashev, A. Haase, J. Neilson, S. Tantawi, S. C. Schaub, R. J. Temkin, and B. Spataro, Prototyping high-gradient $\mathrm{mm}$-wave accelerating structures, J. Phys. Conf. Ser. 874, 012039 (2017).

[6] R. Pompili et al., Energy spread minimization in a beamdriven plasma wakefield accelerator, Nat. Phys. 17, 499 (2021).

[7] M. T. Hibberd, A. L. Healy, D. S. Lake, V. Georgiadis, E. J. H. Smith, O. J. Finlay, T. H. Pacey, J. K. Jones, Y. Saveliev, D. A. Walsh, E. W. Snedden, R. B. Appleby, G. Burt, D. M. Graham, and S. P. Jamison, Acceleration of relativistic beams using laser-generated terahertz pulses, Nat. Photonics 14, 755 (2020).

[8] J. A. Fülöp, S. Tzortzakis, and T. Kampfrath, Laser-driven strong-field terahertz sources, Adv. Opt. Mater. 8, 1900681 (2020).

[9] Z. Tibai, M. Unferdorben, S. Turnár, A. Sharma, J. A. Fülöp, G. Almási, and J. Hebling, Relativistic electron acceleration by focused THz pulses, J. Phys. B 51, 134004 (2018).

[10] S. Turnár, J. Hebling, J. A. Fülöp, G. Tóth, G. Almási, and Z. Tibai, Design of a THz-driven compact relativistic electron source, Appl. Phys. B 127, 38 (2021). 
[11] T. Vinatier, C. Bruni, R. W. Assmann, U. Dorda, F. Lemery, and B. Marchetti, Feasibility study for a THz acceleration experiment on the PHIL accelerator at LALT, J. Phys. Conf. Ser. 1596, 012033 (2020).

[12] M. Fakhari, A. Fallahi, and F. X. Kärtner, THz cavities and injectors for compact electron acceleration using laserdriven THz sources, Phys. Rev. Accel. Beams 20, 041302 (2017).

[13] D. A. Walsh, D. S. Lake, E. W. Snedden, M. J. Cliffe, D. M. Graham, and S. P. Jamison, Demonstration of subluminal propagation of single-cycle terahertz pulses for particle acceleration, Nat. Commun. 8, 421 (2017).

[14] F. Lemery, T. Vinatier, F. Mayet et al., Highly scalable multicycle thz production with a homemade periodically poled macrocrystal, Commun. Phys. 3, 150 (2020).

[15] L. Pálfalvi, J. A. Fülöp, G. Tóth, and J. Hebling, Evanescentwave proton postaccelerator driven by intense $\mathrm{THz}$ pulse, Phys. Rev. ST Accel. Beams 17, 031301 (2014).

[16] E. A. Nanni, W. R. Huang, K.-H. Hong, K. Ravi, A. Fallahi, G. Moriena, R. J. D. Miller, and F. X. Kärtner, Terahertz-driven linear electron acceleration, Nat. Commun. 6, 8486 (2015).

[17] D. Zhang, M. Fakhari, H. Cankaya, A.-L. Calendron, N. H. Matlis, and F. X. Kärtner, Cascaded Multicycle TerahertzDriven Ultrafast Electron Acceleration and Manipulation, Phys. Rev. X 10, 011067 (2020).

[18] D. Zhang, A. Fallahi, M. Hemmer, X. Wu, M. Fakhari, Y. Hua, H. Cankaya, A.-L. Calendron, L. E. Zapata, N. H. Matlis, and F. X. Kärtner, Segmented terahertz electron accelerator and manipulator (STEAM), Nat. Photonics 12, 336 (2018).

[19] N. H. Matlis et al., Acceleration of electrons in THz driven structures for AXSIS, Nucl. Instrum. Methods Phys. Res., Sect. A 909, 27 (2018).

[20] H. Xu, L. Yan, Y. Du, W. Huang, Q. Tian, R. Li, Y. Liang, S. Gu, J. Shi, and C. Tang, Cascaded high-gradient terahertz-driven acceleration of relativistic electron beams, Nat. Photonics 15, 426 (2021).
[21] H. Tang, L. Zhao, P. Zhu, X. Zou, J. Qi, Y. Cheng, J. Qiu, X. Hu, W. Song, D. Xiang, and J. Zhang, Stable and Scalable Multistage Terahertz-Driven Particle Accelerator, Phys. Rev. Lett. 127, 074801 (2021).

[22] F. Lemery, K. Floettmann, P. Piot, F. X. Kärtner, and R. Aßmann, Synchronous acceleration with tapered dielectric-lined waveguides, Phys. Rev. Accel. Beams 21, 051302 (2018).

[23] T. Vinatier, R. W. Assmann, U. Dorda, F. Lemery, and B. Marchetti, Beam dynamics in $\mathrm{THz}$ dielectric-loaded waveguides for the AXSIS project, J. Phys. Conf. Ser. 874, 012042 (2017).

[24] F. Fu et al., Demonstration of Nonlinear-Energy-Spread Compensation in Relativistic Electron Bunches with Corrugated Structures, Phys. Rev. Lett. 114, 114801 (2015).

[25] L. Xiao, W. Gai, and X. Sun, Field analysis of a dielectricloaded rectangular waveguide accelerating structure, Phys. Rev. E 65, 016505 (2001).

[26] M. Aicheler, P. Burrows, M. Draper, T. Garvey, P. Lebrun, K. Peach, N. Phinney, H. Schmickler, D. Schulte, and N. Toge, A multi-Tev linear collider based on CLIC technology: CLIC conceptual design report, Report No. CERN2012-007, 2012.

[27] J. B. García, R. De Maria, A. Grudiev, R. T. García, R. B. Appleby, and D. R. Brett, Long term dynamics of the high luminosity Large Hadron Collider with crab cavities, Phys. Rev. Accel. Beams 19, 101003 (2016).

[28] D. T. Abell, Numerical computation of high-order transfer maps for rf cavities, Phys. Rev. ST Accel. Beams 9, 052001 (2006).

[29] J. Andruszkow et al., First Observation of Self-Amplified Spontaneous Emission in a Free-Electron Laser at $109 \mathrm{~nm}$ Wavelength, Phys. Rev. Lett. 85, 3825 (2000).

[30] B. Shepherd (private communication).

[31] A. L. Healy, Energy modulation of electron bunches using a terahertz-driven dielectric-lined waveguide, Academic theses, Lancaster University, Lancaster, 2020. 